\title{
The Multiplicative Patient and the Clinical Workflow: Clinician Perspectives on Social Interfaces for Self-Tracking and Managing Bipolar Disorder
}

\author{
Michael Jeffrey Daniel Hoefer \\ michael.hoefer@colorado.edu \\ Department of Computer Science, \\ University of Colorado Boulder \\ Boulder, Colorado, USA \\ Lanea Blyss Blackburn \\ lanea.blackburn@colorado.edu \\ Department of Information Science, \\ University of Colorado Boulder \\ Boulder, Colorado, USA
}

\author{
Lucy Van Kleunen \\ lucy.vankleunen@colorado.edu \\ Department of Computer Science, \\ University of Colorado Boulder \\ Boulder, Colorado, USA \\ Priyanka Panati \\ priyanka.panati@colorado.edu \\ Department of Information Science, \\ University of Colorado Boulder \\ Boulder, Colorado, USA
}

\author{
Cassandra Goodby \\ ATLAS Institute, University of \\ Colorado Boulder \\ Boulder, Colorado, USA \\ cassandra.goobdy@colorado.edu \\ Stephen Voida \\ svoida@colorado.edu \\ Department of Information Science, \\ University of Colorado Boulder \\ Boulder, Colorado, USA
}

\begin{abstract}
Personal health informatics are increasingly used in the long-term management of bipolar disorder and other serious mental illnesses. These systems help individuals and members of their support networks track and stabilize important social rhythms. In this study, we presented mental health professionals with nine design concepts for personal informatics systems to support interpersonal and social rhythm therapy. These designs, derived from prior empirical findings about patients' data practices, utilize social interfaces to support the social ecologies critical to relational recovery in bipolar disorder. Designs included features for sharing data in dynamic social support systems, custom variables, commenting, co-tracking, and data-driven action plans. Qualitative analysis of clinicians' feedback yielded design recommendations for mental health informatics, such as supporting all cognitive states of the multiplicative patient and the interactions between these states and patient-clinician interfaces. Diversity in clinical practice also necessitates application flexibility and careful integration with existing workflows.
\end{abstract}

\section{CCS CONCEPTS}

- Human-centered computing $\rightarrow$ Collaborative and social computing; Empirical studies in HCI; HCI design and evaluation methods.

\section{KEYWORDS}

personal informatics, quantified self, self-management, mental health, clinician evaluation, patient-clinician interfaces, serious mental illness, bipolar disorder, social interfaces, social reactivity

Permission to make digital or hard copies of all or part of this work for personal or classroom use is granted without fee provided that copies are not made or distributed for profit or commercial advantage and that copies bear this notice and the full citation on the first page. Copyrights for components of this work owned by others than the author(s) must be honored. Abstracting with credit is permitted. To copy otherwise, or republish, to post on servers or to redistribute to lists, requires prior specific permission and/or a fee. Request permissions from permissions@acm.org.

DIS '21, fune 28-fuly 2, 2021, Virtual Event, USA

(c) 2021 Copyright held by the owner/author(s). Publication rights licensed to ACM ACM ISBN 978-1-4503-8476-6/21/06 . \$ \$15.00

https://doi.org/10.1145/3461778.3461995
ACM Reference Format:

Michael Jeffrey Daniel Hoefer, Lucy Van Kleunen, Cassandra Goodby, Lanea Blyss Blackburn, Priyanka Panati, and Stephen Voida. 2021. The Multiplicative Patient and the Clinical Workflow: Clinician Perspectives on Social Interfaces for Self-Tracking and Managing Bipolar Disorder. In Designing Interactive Systems Conference 2021 (DIS '21), June 28-July 2, 2021, Virtual Event, USA. ACM, New York, NY, USA, 19 pages. https://doi.org/10.1145/3461778.3461995

\section{INTRODUCTION AND BACKGROUND}

Serious mental illnesses (SMI) are estimated to cost over $\$ 200$ billion annually in health care costs, loss of earnings, and disability benefits in the United States alone [33]. Among SMI, bipolar disorder (BD) is a particularly challenging mood disorder with no known cure. $\mathrm{BD}$ has an estimated prevalence of over $1 \%$ worldwide [54], and is characterized by extreme changes in affective state, as individuals cycle between euthymia (steady-state), depression (low mood), hypomania (rising mood), and mania (extremely elevated mood) [29]. $\mathrm{BD}$ is one of the most dangerous classes of SMI, as 1 in 5 individuals with $\mathrm{BD}$ die from suicide [3]. Individuals with $\mathrm{BD}$ also suffer from relationship problems, interrupted career trajectories, and stress associated with the indefinite management of their condition [28].

Pharmacological approaches, by themselves, are generally insufficient in managing BD [24]. A bipolar-specific therapeutic approach, interpersonal and social rhythm therapy (IPSRT), was developed with a purpose of stabilizing important daily rhythms and improving social functioning [21, 23]. IPSRT consists of a union between interpersonal therapy (IPT), which treats unipolar depression by improving interpersonal concerns [39], and cognitive-behavioral techniques to stabilize daily rhythms [24]. IPSRT has been shown to increase the amount of time between affective episodes and the regularity of social rhythms [22], as well as to reduce suicide attempts [71].

An important behavior-based component of IPSRT includes tracking and stabilizing everyday activities such as light exposure, sleep-wake routines, mood state, and social rhythms, which can result in pronounced functional improvements and minimized symptom severity for people with BD $[23,55]$. Clinical guidelines 
therefore encourage incorporating daily self-monitoring into condition management [36]. Furthermore, self-tracking can provide a sense of agency for people whose disorder makes them feel out of control [58], help individuals establish and maintain a sense of identity as their condition evolves [32], and promote mindfulness and commitment to behavioral goals [49].

Within HCI research, personal informatics (PI)-systems that help individuals collect and explore data about themselves-have been shown to be well-suited to supporting self-tracking in BD (e.g., $[4,48,50,59,60])$. However, previous work highlights that individuals with $\mathrm{BD}$ tend to create their own homegrown, customized solutions for managing their condition [49], largely due to inadequacies or mismatches between BD self-tracking data practices and the functionality of existing technologies [60]. While self-tracking in some contexts has been perceived as a burden or unsupportive of "illness work" [47], the clinical support provided through contexts of CBT and IPSRT motivate the development of self-tracking systems for managing BD.

Traditional research in the self-tracking literature focuses on the design of PI systems to support the lived experience of individuals [17], as well as their progression through different stages of self-tracking [42]. However, the interpersonal components of IPRST motivate the inclusion of one's social support network in the design of PI systems. The "IP" in IPSRT focuses on problem solving in and maintenance of a patient's relationships. This approach aligns with the notion of "relational recovery," acknowledging the role that family, friends, antagonists, and even organizations and cultures play in coping with and managing long-term SMI [69]. Considerations for these interpersonal contexts in which the self-tracking technology is situated have only recently begun to take hold in the self-tracking literature (e.g., [59, 60]). Initial work on these social PI practices, such as sharing data amongst close contacts, have uncovered a variety of needs of individuals with BD that are currently not being met by existing PI systems, such as supporting the social contexts where the data are used.

Previous empirical studies $[49,60]$ motivate a number of design requirements for a self-tracking system to address the shortcomings of existing tools: an ability to manage dynamic relationships and roles, transparency, supporting long-term condition management, supporting social relations, and a diversity of individual tracking preferences. One might imagine these diverse, empirically grounded design requirements being integrated together in the design of a single system in the following scenario, which reflects the voice and representative concerns of an individual with bipolar disorder, constructed as a composite of participant quotes from these prior studies:

I want an app that lets me track whatever I want, and share it with whomever I want, whenever I want. And those people change all the time-I've been on and off with my partner for the last three years. Mood, sleep, shopping, sex, whatever I want to track. I want to be able to control who sees what data... and it had better be secure, because if data like this leaks out, if might cost me the ability to get the job that I want because of stigma against mental health issues. Nothing too fancy either, I've got enough complications in my life. And if it could give me some data about which medication works best, that would help. I guess it should let me share data with my doctor too, to help with these medication decisions. And it'd be nice if my doctor could understand what my life is like outside of our rushed office visits. Also, my parents live far away, but they're still a big part of my life, so it should include them too. They like to be updated on what I do, and if they could see that things were fine maybe they'd stop bugging me so much.

The social support context in which this application might be used motivates the necessity of including design perspectives not only from family and friends, but also from clinicians. Clinicians prescribe medication and administer various types of therapy, which both play a significant role in the long term management of most SMI, including BD. However, current PI systems for bipolar fail to address clinical interfaces in their design, and instead rely on document exports to support the clinical workflow. In addition, design considerations for the inclusion of self-tracked data in a clinical setting have been relatively unexplored. Studies with clinicians for self-tracked data on physical health uncovered clinical concerns, such as suspect data believability [77] or clinician workload and information overload concerns $[16,45]$. However, the use of self-tracked mental health data in clinical contexts has yet to be fully explored.

\subsection{Statement of Contributions}

In this research, we developed nine design concepts for a flexible self-tracking application for managing bipolar disorder, based on requirements from prior empirical studies of the data practices of individuals with BD. We gathered a clinical perspective on these designs through interviews with 17 mental health professionals. A qualitative analysis of the feedback resulted in insights for the design of personal informatics systems both for BD and other SMIbut also for general PI and self-tracking systems embedded in social contexts.

Besides providing concrete guidance on the clinical suitability of specific application features represented by our design concepts, our inductive analysis of clinicians' feedback highlights the need to support the different, unique cognitive states that an individual with bipolar disorder may be in at a given point in time-the multiplicative patient. System design can take advantage of these different user states by supporting intra-individual communication, and preventing intra-individual conflict. We also suggest ways in which PI systems can be used flexibly by diverse clinicians to accommodate their clinical workflow, workload, and expectations.

\section{RELATED WORK}

The widespread adoption of the smartphone has led to an interest in developing mobile health (mHealth) applications for managing and supporting SMI [68]. A variety of considerations for designing applications in this space have been previously described [16], and include handling data privacy, supporting continued engagement [7], and integration with clinical workflows [11]. In the case of bipolar disorder, previous studies have shown that individuals with $\mathrm{BD}$ are receptive to mobile health interventions involving textbased symptom monitoring [9], and that remote self-monitoring 
of mood has a high correspondence with standard psychological instruments used to assess mania and depression [6]. Using technology to support long-term treatment and management of BD moves towards the notion of the digital phenotype, where active and passive data about a condition are tracked and used in clinically relevant ways [34]. Informatics systems that support digital phenotyping can help improve clinicians' ability to accurately diagnose and manage treatment of health conditions, as well as better understand the 'lived experience' of patients [64].

A variety of digital tools have been developed to support individuals with BD [26]. Web-based tools include forums for users to connect with others to share advice [41], educational platforms to learn strategies for condition management [70], and general mood and daily rhythm tracking [43]. Most similar to the present study is the development of systems for collecting data for symptom detection, such as MoodRhythm [48, 75] and MONARCA [4], which collect both objective and subjective data. MONARCA and MoodRhythm both predefine a set of measures to be tracked by users [5], and do not incorporate custom variables. While both systems provide affordances for feedback and reflection on selftracked data, MONARCA also provides a five-day mood-prediction forecast using machine learning [25]. However, these systems do not support the social ecologies shown to be effective in supporting long-term management of BD [60], motivating the evaluation of new social interfaces for self-tracking and bipolar disorder.

An important social practice for personal informatics systems is the sharing of health data between patient and clinician [78]. An open research area is how to best integrate patient-generated health data with clinical workflows [45]. Surveys and interviews with clinicians have highlighted how the use of self-tracked data in clinical settings is inherently collaborative, and can be used to navigate tensions between patient and clinician [12]. However, a vignette role-play study with physicians highlighted the necessity for clinicians to evaluate data quality, completeness, and even patient motivations for engaging in self-tracking [77]. In addition, a series of interviews with a variety of medical professionals described common barriers to integrating data in various clinical settings, including challenges in data representation and customization from patient to patient [78]. While this study did include the perspectives of two mental health professionals, the bulk of the feedback was from physical health professionals. A shared finding from these studies is the importance of the interfaces with which clinicians engage with patients and their self-tracked data. Given the subjective and often socially-influenced nature of self-generated mental health data, an exploration of these social interfaces in a mental health clinical setting is warranted.

The present study builds on a series of multi-stakeholder user centered design studies published in recent years, including interviews uncovering effective self-tracking practices for those with BD [49], a study uncovering the ways in which interpersonal contexts support long term condition management [60], a visual exploration of individuals' lived experience [74], and a study understanding concerns for privacy and control of data sharing in this context [66]. In light of this previous work, we have identified at least three key challenges that must be addressed in future designs of self-tracking applications for collaboratively managing BD:
- Design Challenge \#1 (DC1): Allowing for dynamism in relationships and roles expected with long-term use of the system;

- Design Challenge \#2 (DC2): Allowing for fine-grained management of data disclosure between individuals with SMI and members of their support networks; and

- Design Challenge \#3 (DC3): Allowing individuals flexibility in the variables that they self-track to more accurately represent their lived experience of serious mental illness.

Our current study aims to evaluate design concepts which are possible solutions to these stakeholder-driven challenges to see whether they can be integrated into clinical practice. This is an essential part of a long-term, iterative user-centered design research process (see $[20,72]$ ) in which we will design and implement a smartphone-based, collaborative self-tracking application for the management of bipolar disorder-with stakeholder involvement throughout the process.

\section{DESCRIPTION OF DESIGNS}

The general purpose of the proposed application is for individuals with bipolar disorder to collect data about their lives and share it-safely and appropriately-with members of their social support system. The designs presented in this paper draw on previous work engaging with individuals with bipolar disorder regarding visualization [74], transparency [66], and interactions among members of social support networks [60]. Out of this research arose a variety of patient-centered designs that were prototyped as wireframes using Figma [76]. While many of the designs are presented using mood state as a sample variable to be tracked, the same interfaces could be used to track other variables relevant to long-term management of $\mathrm{BD}$ (mentioned by the clinicians interviewed), such as sleep/wake times, irritability, suicidality, food consumption, physical activity, social activity, sexual activity, and spending, among others.

The designs consist of (1) adding and removing people over time, (2) displaying one's overall state, (3) data sharing via aggregation, (4) data sharing via discrete shares, (5) data commenting, (6) tracking custom variables, (7) dynamic tracking methods, (8) co-tracking, and (9) automated action plans. In this section, we briefly present each design concept and its rationale.

\subsection{Design One: Adding and Removing People Over Time}

Changes to an individual's social support system-even those that seem "permanent," like family relations-are inevitable [60], and these changes need to be supported by self-tracking systems for SMI intended for long-term use (DC1, DC2). For example, a patient may have a falling-out with a family member, make a new friend at a support group meeting, change jobs, or switch to a new clinician. Figure 1(a) shows a design that allows patients to add or remove individuals from the application and provide others access to historical data for different tracked variables. In this design, newly added users, such as a new clinician, receive a corresponding notification on their devices to make them aware of the new link established through the app. 


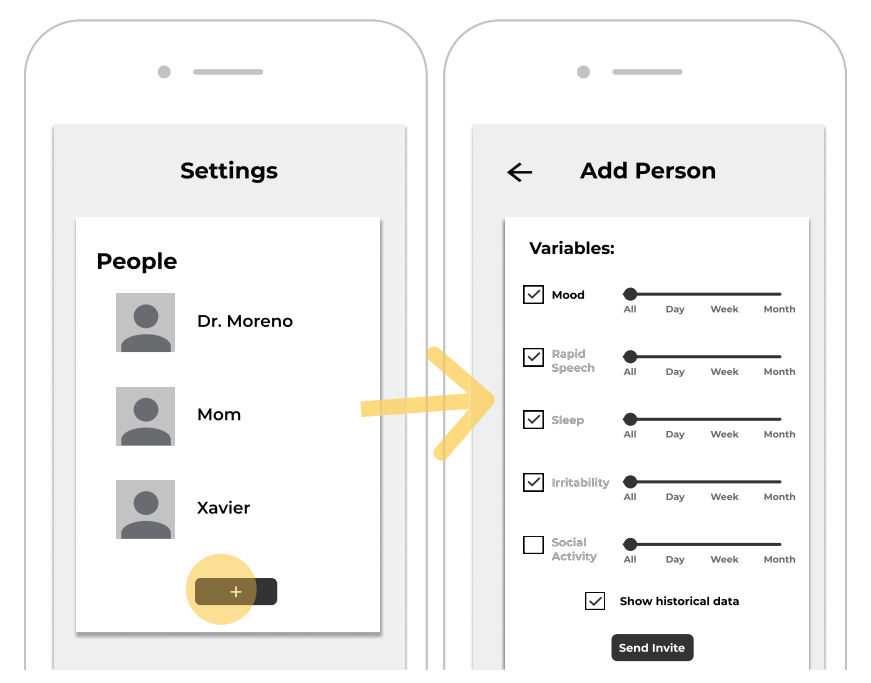

(a)

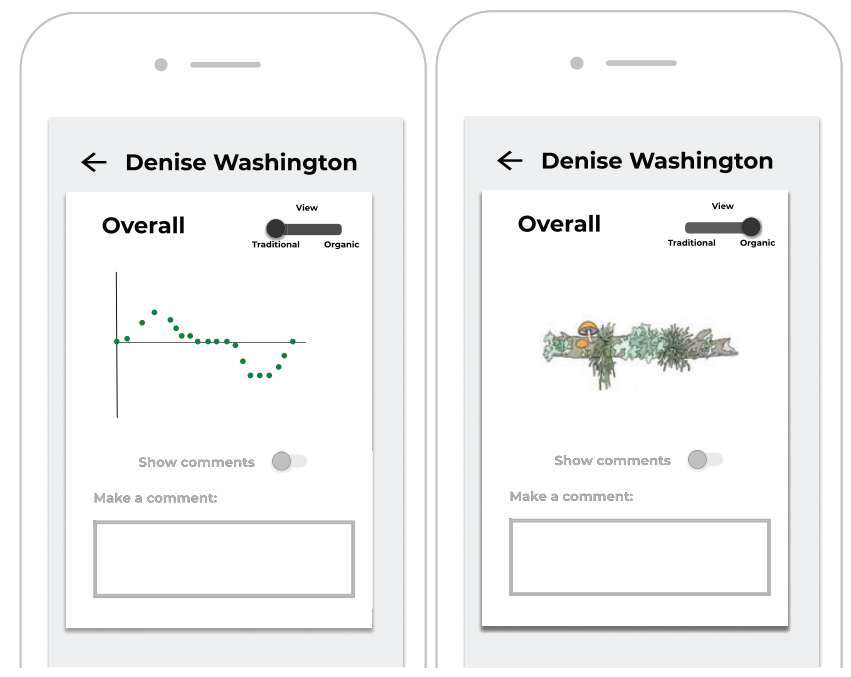

(b)

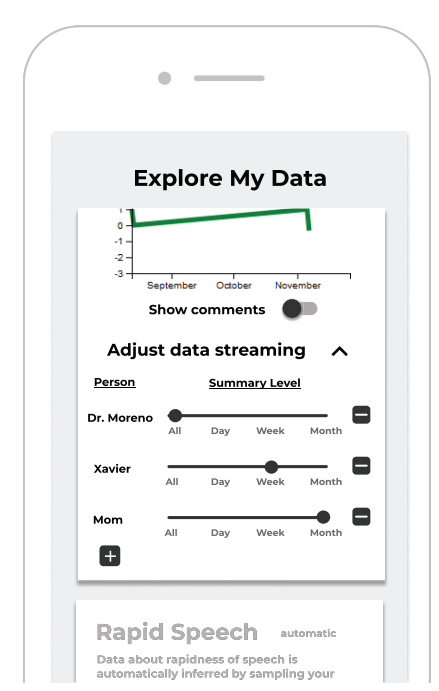

(c)

Figure 1: A montage showing the most relevant excerpts of the proposed mobile app designs we showed to clinicians in our study. In these wireframes, patients' phones are unshaded (white) and clinicians' phones are shaded (light blue). (a) Design One: System users can be added and removed. (b) Design Two: Variations of designs for displaying overall state-left: numerical display; right: organic display. (c) Design Three: Controlling the level of aggregation of data sharing.

\subsection{Design Two: Displaying and Sharing Overall State}

Although individuals often self-track a variety of variables, there is a desire to view one measure that represents how an individual is doing, overall (DC 2, DC 3). In this feature, we create an index that combines all of the variables into a representation of overall state. The left side of Figure 1(b) shows how this index might be displayed in a numeric format.
Previous interviews highlighted that numerical quantifications of one's overall state do not always reflect the lived experience of those with bipolar disorder [74]. Individuals may prefer to look at a natureor circular growth-inspired visualization as it can better represent their experience with bipolar disorder, where linear growth is not always possible. This visualization can also help support system members empathize with their condition. In the right side of Figure 1(b), we show an example how an organic visualization developed as part of this prior research might be used in this capacity; this 

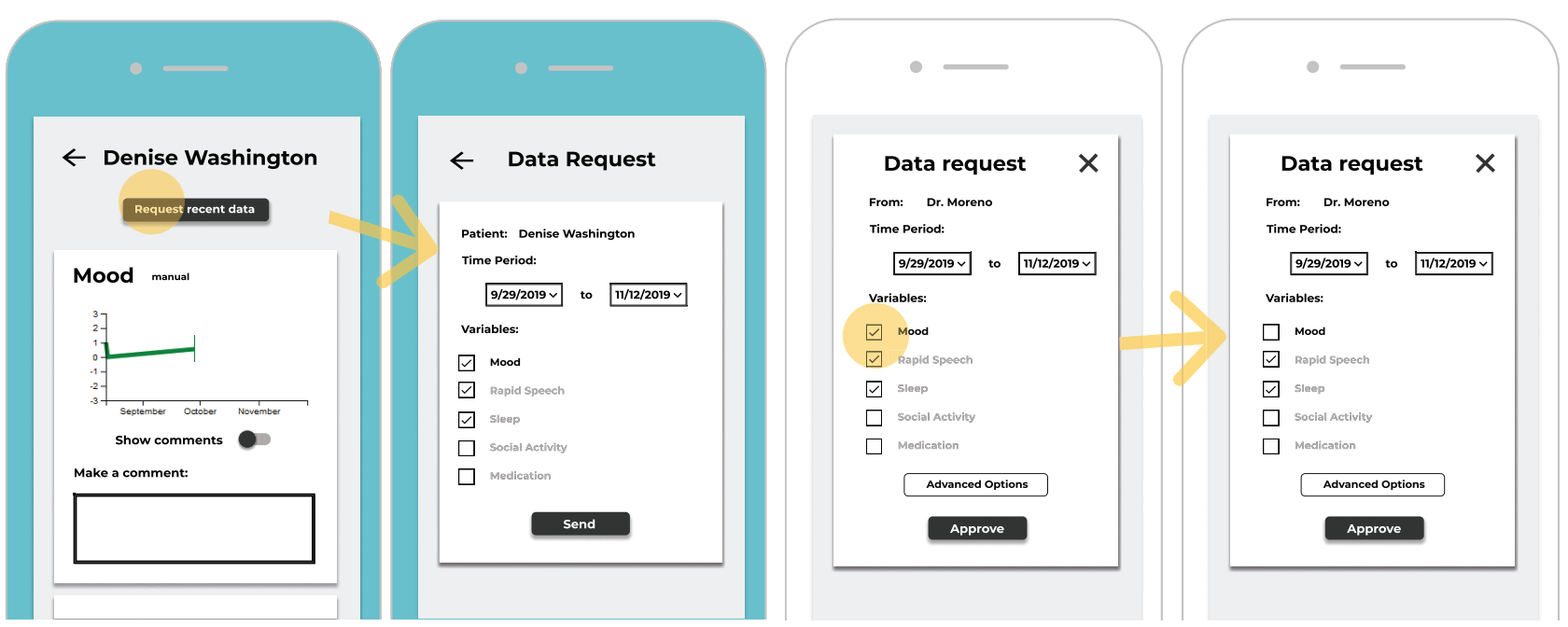

(a)

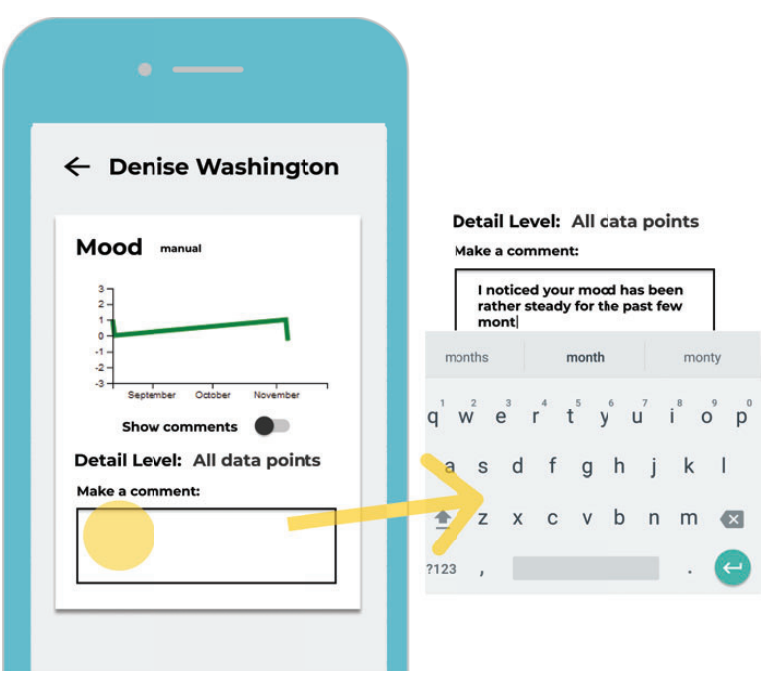

(b)

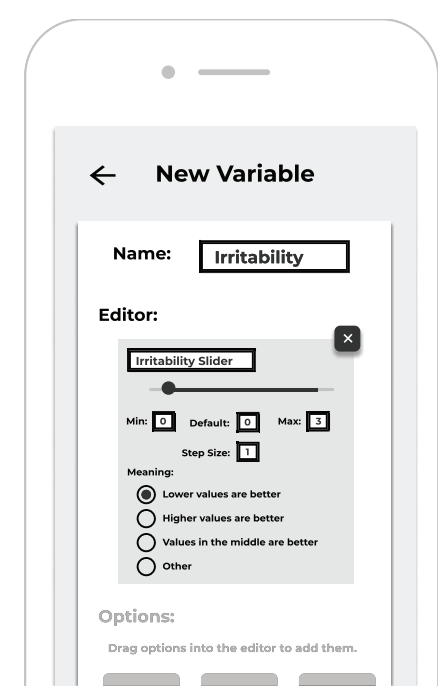

(c)

Figure 2: The most relevant excerpts of the proposed mobile app designs (continued). In these wireframes, patients' phones are unshaded (white) and clinicians' phones are shaded (light blue). (a) Design Four: Data sharing via discrete shares-left: data is requested; right: individuals create a privacy-preserving adjustment. (b) Design Five: Individuals-here, the clinician-can add comments to the data. (c) Design Six: Custom variables can be configured.

particular visualization is one of a fallen log with various plants and mushrooms growing on it. The life on the log will grow or decay based on the user's overall state. The design of the particular organic visualization to be implemented is a topic of current research [74]. All users can toggle between the traditional numerical view and this organic data view.

\subsection{Design Three: Data Sharing via Aggregation}

Prior work revealed many instances of individuals with BD intentionally sharing personal data with individuals in their microlayer [60]. This sharing was motivated by a desire to build mutual awareness and empathy around the experience with $\mathrm{BD}$ and to improve loved ones' ability to notice lapses in effective condition management or crisis warning signs. Respondents shared data with clinicians primarily in order to aid in decision making related to their treatment. 


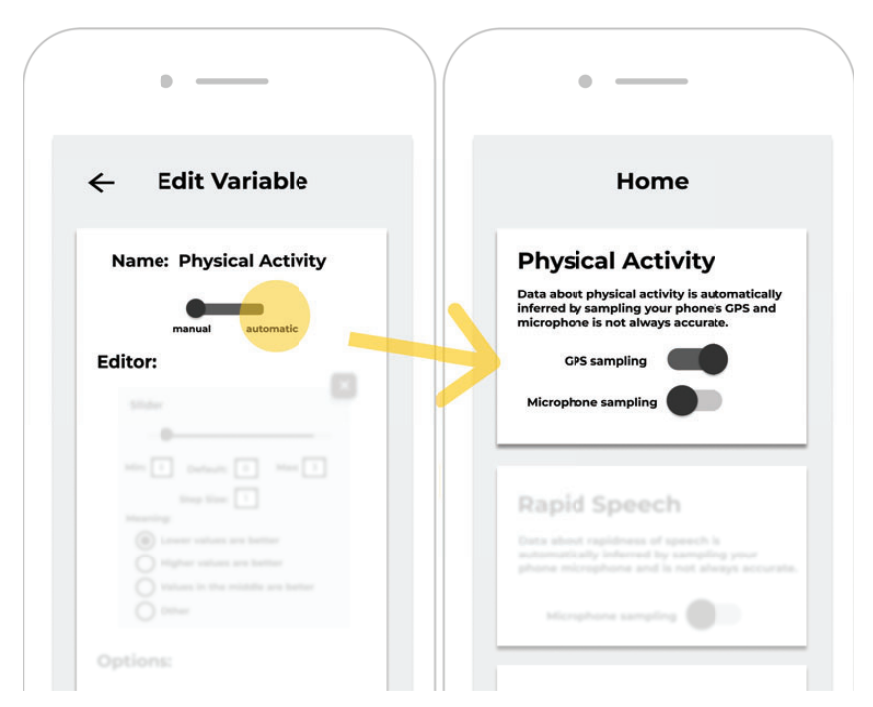

(a)

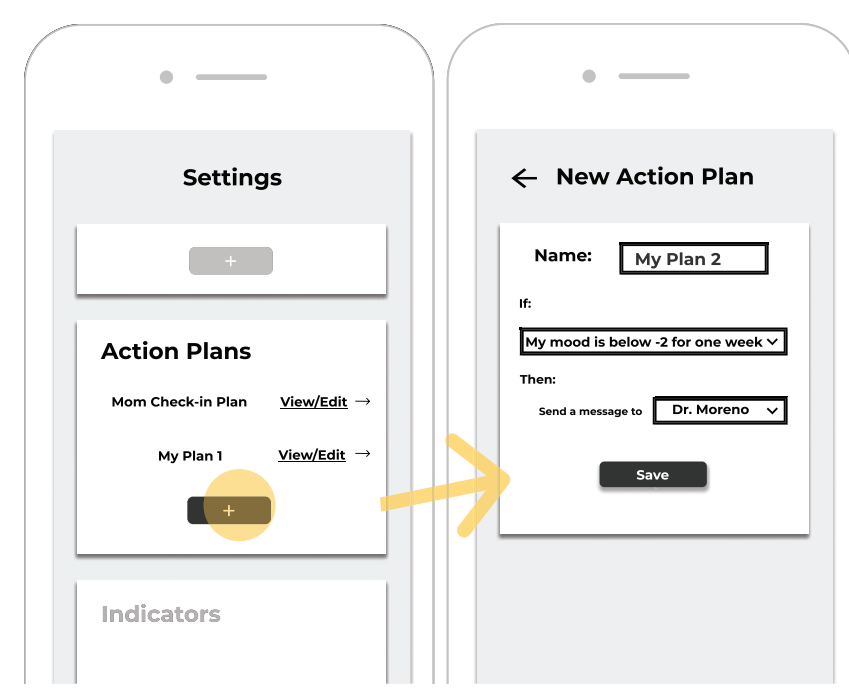

(c)

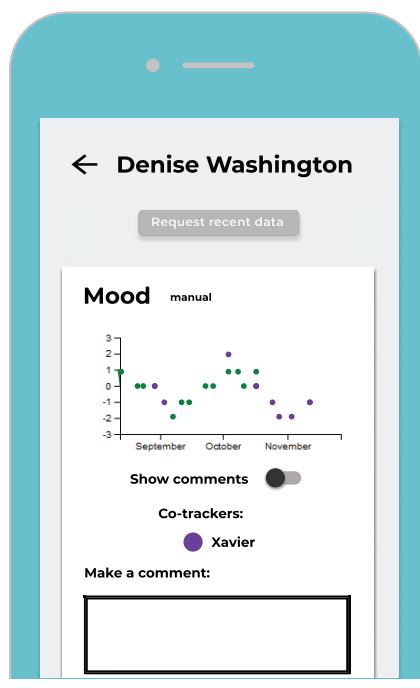

(b)

Figure 3: A montage showing the most relevant excerpts of the proposed mobile app designs we showed to clinicians in our study. In these wireframes, patients' phones are unshaded (white) and clinicians' phones are shaded (light blue). (a) Design Seven: Individuals can switch between automated and manual tracking. (b) Design Eight: Data streams from multiple cotrackers are visible on the same chart. (c) Design Nine: Creating action plans-left: an individual creates an action plan to automatically notify a clinician if the tracked mood is below -2 for one week; right: a clinician is notified that an action plan has been triggered.

However, many individuals were concerned about unintentional disclosure of personal data. In some cases, they did not want to unnecessarily worry their loved ones or burden them with information about their condition. In more extreme cases, they worried that details of their condition might be exposed to unsympathetic employers or individuals from their past with whom they had negative or abusive relationships. Possible negative consequences of leaking sensitive health data were the most common reasons noted by individuals for avoiding self-tracking altogether.

Overall, we saw that individuals with BD have diverse perspectives regarding disclosure and complex criteria when determining which data will be shared, with whom, and how. Supporting social PI practices in the SMI domain requires nuanced design and engineering solutions that go beyond simply enabling multi-user 
account access in self-tracking apps or enabling social media-based personal data sharing.

To this end, we developed two schemes to support data sharing with fine-grained transparency controls (DC 2). The first design utilizes aggregation to control transparency. In this scheme, data is continuously shared with individuals, and patients have the ability to adjust the level of aggregation with which data is shared. Figure 1(c), for example, shows that the patient is giving their therapist (in this example, Dr. Moreno) access to all data points, while their spouse (Xavier) receives weekly summaries, and their mother receives monthly summaries. The level of aggregation an individual has access to is also clearly indicated on the recipient's screen.

\subsection{Design Four: Data Sharing via Discrete Shares}

An alternative scheme for sharing data conceptualizes sharing as occurring via discrete "requests" for and "shares" of specific data (DC 2), which has been shown to be valuable in instances in which "an individual with BD feels like their behaviors, symptoms, or needs are visible but being dismissed" [66]. In the example shown in figure 2(a), a clinician is requesting recent data from a patient before an appointment. The request consists of a set of variables to be shared across a date range. The left side of Figure 2(a) shows the patient's interface for responding to a data request. The patient can view and modify the request to preserve privacy. In the right side of Figure 2(a), the patient modifies the request by choosing not to share the "Mood" variable. The clinician is notified that the request has been modified but is not shown the particular modifications in order to preserve patient privacy. However, data-sharing omissions (in general) can be discussed with the patient.

\subsection{Design Five: Data Commenting}

Previous interviews highlighted that individuals with bipolar disorder often wanted to call attention to some behavior, symptom, or need, or note an event [60]. To support this, we propose a design to enable both patients and their support system members to make comments about the data (DC 2), similar to how clinicians and patients may annotate data during live office visits [53]. This feature may also help to reduce the "systematic distortion" of meaning of diagnostic labels often present in patient-clinician interactions [2], by adding valuable context to numeric representations of the lived experience and allowing the clinician to actively respond to changes in the patient's state. Figure 2(b) shows the interface being used by a clinician to add a comment. In the patient's view, comments are time-stamped and displayed directly on the chart to provide context. Comment visibility can be toggled on and off.

\subsection{Design Six: Tracking Custom Variables}

Prior research has identified that individuals managing SMI are particularly vulnerable to feeling judged, flawed, and isolated when confronted with normative PI measurement scales that do not align with their own mental models that they use to gauge and make sense of their condition [49]. For example, it might be challenging for someone with SMI to understand how to self-assess mood on a numerical scale.
Instead, individuals had different ways of keeping track of symptoms and triggers. While the application may have a set of default variables, we also provide the capability for individuals to customize how they self-track (DC 3), providing the flexibility that is often present in patient-driven observations of daily living (ODL) [67]. In a clinical setting, individuals would discuss with their providers how to best set up custom variables [12]. Figure $2 \mathrm{cb}$ ) shows how patients can add a new custom variable, specifying the range of numerical options along with which values are 'better.' There is also an option to utilize binary checkboxes or photo uploads to track custom variables.

As patients may be tracking the same variable using different methods, clinicians may see different scales and methods of tracking across different patients. For example, mood might be displayed on a -3 to +3 scale for some patients, while others use photo uploads to represent their mood.

\subsection{Design Seven: Dynamic Tracking Methods}

We learned that patients may change how they manage their condition over time, and clinicians may change their focus of treatment over time [2]. For example, an individual may be more engaged in hands-on tracking immediately after being diagnosed with bipolar disorder to identify triggers, and then track less over time. To support this dynamism, we propose a design feature that allows individuals to switch between manual and automated tracking of certain variables (DC 3). The application could perhaps take advantage of a smartphone's GPS sensor to track physical activity, or a microphone to identify patterns of speech that may indicate a changing affective state [30].

In Figure 3(a), the individual switches from manually tracking physical activity on a scale of zero to three, to automated tracking using GPS sampling. This switch is clearly noted when displaying the data, as the scale will be different between manual and automated tracking.

\subsection{Design Eight: Supporting Co-Tracking of Variables}

We learned that people with bipolar disorder rely on close members of their support system to help them keep track of how they are doing [49]. These close relations may take over tracking duties if the person was unable to track, for example, if they were having a manic episode. This design provides explicit support for this kind of distributed co-tracking across multiple accounts, which is an activity that would otherwise require sharing access to the same spreadsheet or physical smartphone device, which opens up additional information sharing concerns and is pragmatically impossible when the social network members are not co-habitating (DC 1, DC 2, DC 3). Prior diary studies have indicated a high degree of empathic accuracy in partnerships [13, 73], including awareness of suicidality and depressive symptoms [51], suggesting co-tracking is an effective method for interpersonal monitoring of well-being.

For example, consider a case in which a patient with $\mathrm{BD}$ adds their spouse as a co-tracker for mood. As the spouse tracks data for their partner on their own phone, this data is noted on the visualization interface, with data points color-coded based on who 
submitted each report (Figure 3(b)). It is also possible for both the patient and their co-tracker to track the same variable on the same day, allowing for comparing multiple perspectives on the same variable.

\subsection{Design Nine: Automated Action Plans}

Individuals with BD often set up "action plans" with members of their support system related to specific triggers. For example, an individual may ask their spouse to call their therapist or take them to the hospital if they see the individual engaging in a particular behavior. To support this functionality within the application, we propose automated action plans that are triggered based on the various data currently being tracked (DC 1, DC 2, DC 3). This feature provides digital infrastructure for the last stage in the sixstage workflow (decide on a plan or action), as modeled by West and colleagues [78].

On the left of Figure 3(c), we show a patient setting up an action plan which is triggered if the tracked mood is below a level of -2 (reported on a numerical scale) for one week. The action plan is set up to automatically notify the therapist if the criteria are triggered. The right side of Figure 3(c) shows what happens when the clinician is notified through the application about the action plan being triggered, prompting him or her to take action on the patient's behalf.

\section{METHODS}

We recruited and interviewed $N=17$ mental health professionals from the Denver metro area to participate in a semi-structured interview to evaluate the designs. We defined a mental health professional following the characterization by the National Alliance on Mental Illness-as a professional who can provide assessment and therapy and/or prescription and monitoring of medication related to mental health and is specialized in mental health care [63]. Our inclusion criteria was therefore limited to psychologists, counselors, clinical social workers, psychiatrists, and psychiatric mental health nurse practitioners. We specifically recruited mental health professionals who had experience treating people with bipolar disorder. Participants were recruited through contact information posted on publicly available websites, postings on mental health professionally oriented online forums, snowball recruiting, and prior connections with the research team.

Participants provided consent for participation and recording via DocuSign. Interviews were approximately one hour long and performed over the Zoom video-conferencing application. The designs presented above were curated into a Google Slides presentation that was concurrently opened by both the researcher and participant during the interview.

The interviewer first showed the participant two overview slides about the basic idea of the application. Participants were instructed not to focus on low-level details like font size and color, but rather consider if the particular design could be incorporated into clinical practice. The interviewer presented each design to the participant and asked for qualitative and quantitative feedback on each design.

Quantitative feedback was gathered via a "stoplight" style interface. Participants were instructed to mark one of three colors for each design. Selecting "green" indicates the participant thinks
Table 1: Licensure information of participating clinicians

\begin{tabular}{lc}
\hline Licensure & $\begin{array}{c}\text { Number of } \\
\text { Participants (\%) }\end{array}$ \\
\hline M.A. Clinical Psychology & $2(12 \%)$ \\
Ph.D. Clinical or Counseling Psychology & $4(23 \%)$ \\
Psychiatric Nurse Practitioner & $3(18 \%)$ \\
M.D. or D.E.O. (practicing Psychiatry) & $5(29 \%)$ \\
Clinical Social Worker (LCSW) & $2(12 \%)$ \\
Professional Counselor (LPC) & $1(6 \%)$ \\
\hline
\end{tabular}

the design would be beneficial, "yellow" indicates the participant is unsure if the design would be beneficial or the participant has some minor concerns about the design, and "red" indicates the participant has a major concern or thinks the design may be harmful. A similar visual feedback system has previously been used to display cybersecurity risks [61].

Qualitative feedback was gathered via verbal comments throughout the study, which was recorded using the Zoom "Record Meeting" feature. Participants could also leave written comments on specific parts of the design using the Google Slides commenting feature, and could ask questions at any time. After the designs were presented, additional questions were asked about the application as a whole. Participants were prompted to provide a ranking of the top three most useful design features, discuss major concerns about the designs, and provide other general feedback about the application. Lastly, participants were asked "do you think an application like this one should be built?"

Quantitative stoplight data was aggregated to generate summary statistics of clinician responses. Designs with more than one wireframe had multiple ratings per clinician. Interview transcripts were analyzed using inductive thematic analysis [15], utilizing MaxQDA software [27].

Finally, we conducted a post-analysis member check [44] with our participants to ensure the accuracy of the reporting and to provide an opportunity for participants to provide additional feedback based on their (anonymized) professional colleagues' responses and observations. In our member check, we provided an early draft of the results and discussion, after the data had been synthesized [8]. We received responses from $8 / 17$ participants, with no suggested changes besides a correction to one participant's professional title.

\section{RESULTS}

\subsection{Participant Demographics}

The licensures of the participants are presented in Table 1. Clinician experience ranges from one year to 35 years, with an average across clinicians of 14.7 years. The number of patients seen per week ranged from 2 to 40, with an average of 18.4 patients. All of our participants had experience treating individuals with bipolar disorder, although from different clinical approaches. The practicing psychiatrists we interviewed tended to focus on symptom assessment and treatment with medication and tended to see patients less frequently. The psychologists, counselors, and social workers tended to provide ongoing therapy, independent of prescribed medication(s), and may see their patients more frequently. 
This diversity in sampling enabled us to explore how self-tracking technology could fit in with various facets of professional mental health support that an individual with BD may seek.

\subsection{Clinician Evaluation of Design Proposals}

Stoplight ratings for all participants were aggregated for each design, and are presented in Table 2. Overall, 327 ratings were collected, with $69 \%$ green, $27 \%$ yellow, and $4 \%$ red.

5.2.1 Design 1: Adding and Removing People Over Time. Feedback from the first design was generally positive, with $88 \%$ green responses. Clinicians were generally supportive of individuals having the freedom to add and remove whomever they wanted: "I like that people can be added and removed over time because that's super relevant for patients." -Clinician 7

The primary concerns ( $12 \%$ yellow responses) involved individuals acting differently during a manic episode, potentially removing everyone from the access list in the application: "Sometimes when they [individuals managing bipolar disorder] are getting really elevated and are manic, this might be a time where they actually take everyone off [the application]." -Clinician 11

In this sense, the act of removing an individual from the application may, in itself, be therapeutically relevant and worthy of note. This concern informed a design modification where individuals are notified if they are removed from the application. The individual can then take action if the removal from the application was a surprise indicative of a potentially changing mood state.

During interviews, the following additional design modifications were suggested by clinicians:

- Require at least one person be added on the application.

- Create an undo button to reverse changes made to permission settings.

- Store support system members contact information, and provide an option to share this information with clinicians in the case of emergency.

5.2.2 Design 2: Displaying Overall State. Discussions around aggregating individual variables into an overall representation of an individual's state uncovered a variety of clinical considerations. Clinicians did see value in being able to quickly assess a patient's overall state, to save time and view the "big picture:" "I want to be able to quickly look at how this patient's doing so I can understand it as opposed to sifting through like 12 different scales." -Clinician 1

However, only $38 \%$ of the ratings for this design were green ( $56 \%$ yellow, $6 \%$ red). Multiple clinicians brought up considerations and cautions for how the different tracked variables could be aggregated into a single measure. One red rating was for the possibility of combining variables in a way that hides trends that are only visible when looking at a single variable, or looking at finer-grained time epochs. In addition, individuals may be tracking different variables and have differences in what an overall state might represent: "If you were manic in the morning and depressed at night and then if you're averaging them, it's going to look like the person was fine." -Clinician 1

Additionally, there was a concern that attempting to generalize or to compute the value of these variables relative to a broader population could also be a challenge, a problem that resonates with the known challenge of data normatization in the context of BD [74]: "I just don't know how you would then take that information and be able to write an algorithm that would effectively combine it so that you could work across a larger population of individuals." -Clinician 1

Clinicians also suggested developing a measure that goes beyond simple averaging of variables, such as counting "points" when particular variables are outside healthy ranges for certain amounts of time. A method like this could draw from statistical process control in industrial settings, such as the Western Electric Alarm Rules, which contain criteria for when measurements outside the normal range indicate a significant change in the process being measured [14]. In addition, clinicians suggested that the application clearly state which variables go into the aggregation, as well as provide users the option to "break down" the aggregation into the component variables: "II] would want to be able to figure out if those trends are related to one particular facet of their symptoms." -Clinician 17

Clinicians also had mixed views about the use of the organic visualization. The second red rating for this design was due to the potential for the organic visualization to cause distress-for example, if the visualization depicted death ("I'm doing so poorly my mushroom died" -Clinician 16). While clinicians tended to feel that an organic visualization would take more time to interpret ("It's just not as useful for me...thinking from the mindset of a clinician and just need for efficiency and expediency" -Clinician 5), it would also help facilitate subjective conversations with the patient. In addition, it would be helpful for their patients to communicate to their support system members: "There are some patients that would much rather have a more graphical, visual representation of how they're doing and feel it communicates better. So again, the idea that you have both the organic and the traditional toggle I think makes it work." -Clinician 13

Lastly, clinicians also mentioned that patients may want to have a choice as to the visual representation of their overall state, motivating the inclusion of a variety of graphical representations.

During interviews, the following additional design modifications were suggested by clinicians:

- Display separate variables representing "mania" and "depression" and display them on the same chart.

- Enable users to select which variables they want to have included in the calculation of overall state.

- Create a binary variable representing if the patient is currently tracking or not, which is clinically relevant.

- Place components of organic imagery on the numerical graph.

5.2.3 Design 3: Data Sharing via Aggregation. Clinicians were supportive of an individual's right to disclose their own data, and highlighted how this level of control would enable patients with bipolar disorder to navigate a variety of dynamic relationships (85\% green responses): "If you are a patient, you want to control how much data other people are seeing. It's a big deal for them to allow others to see any level of their data... giving them that extra sense of control, I think, is important." -Clinician 13

However, clinicians also stressed the importance of having the correct level of aggregation available for the right individuals at the right time; as a result, 15\% flagged this design as being yellow or 
Table 2: Frequency of colored stoplight feedback for each design

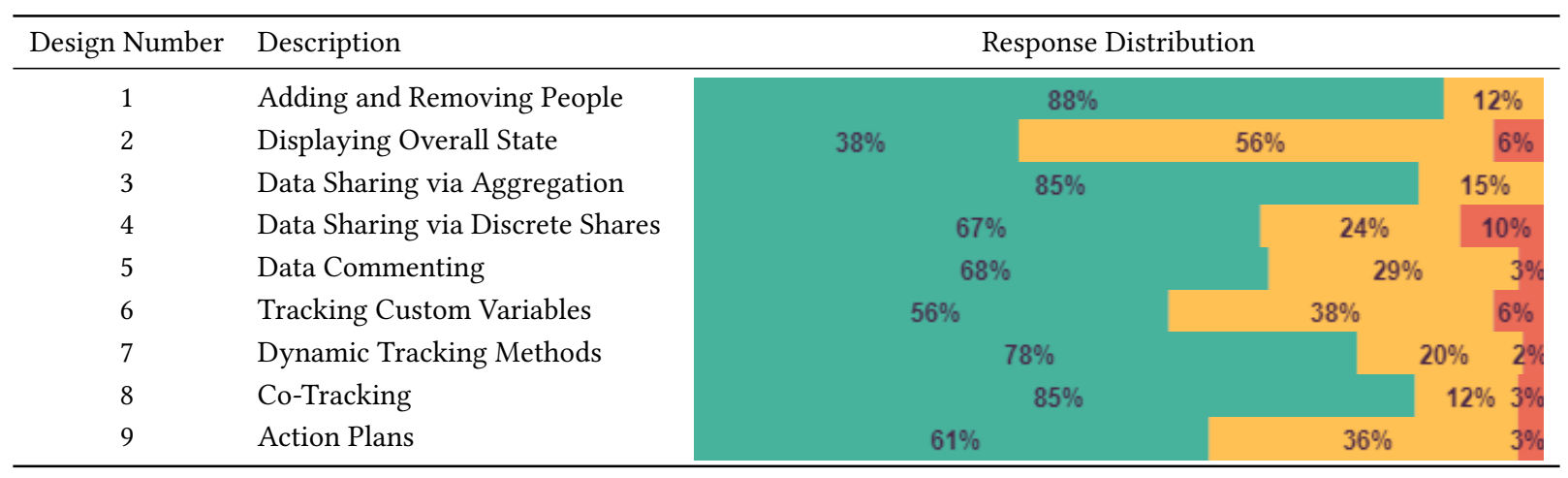

"caution." It was particularly important for clinicians to be able to see a fine-grained level of detail in the visualization: "I can see how it might be helpful for a distance support person, like an adult parent who's not living with them, to just give them an overall. But if they're in crisis, they really need to see something in the acute moment, rather than, you know, the month summary down the line." -Clinician 9

Clinicians liked that the patient was able to adjust the level of aggregation, but wanted to ensure that, as their clinician, they would be able to see a high enough level of detail to make treatment decisions: "If I were that person's provider, I would want to see everything." -Clinician 4

During interviews, the following additional design modifications were suggested by clinicians:

- Enable individuals to see which variables they do not have access to.

- Send a text reminder to the patient asking them to confirm existing data access permissions every so often.

5.2.4 Design 4: Data Sharing via Discrete Shares. Discrete shares provide individuals with a finer-grained level of control as compared to continuous sharing, as they can control the audience of even a single piece of data (such as a single mood rating). Clinicians were primarily concerned ( $24 \%$ yellow, $10 \%$ red ratings) with the level of effort required to manage these detailed permissions and with the risk of the individual omitting critical data from the clinician's datastream: "The discrete shares of data sounds, for me as a clinician, really time consuming... if I have any time, I'm going to be reviewing the chart. I'm not going to pull up my phone app probably and ask them for consent." -Clinician 10

Clinicians also expected the workload of managing permissions to be prohibitive for the individual sharing data, especially if the individual was in a manic state: 'Somebody who's really manic is probably not going to take the time to look through these individual things." -Clinician 9

In addition to workload issues, clinicians were also concerned with a risk of incomplete data. Not only could the application facilitate the hiding of data, this data omission could be clinically misleading: "I wouldn't want to promote some patients trying to represent doing better than they are... the bottom line goal is in the service of their [the patient's] stability and treatment and that it's not misrepresenting how they're doing." -Clinician 14
Some mentioned that if the data was not complete, it may not even be worth looking at ("If I'm not able to trust that it's the full dataset, is it even worthwhile?" -Clinician 5). Other clinicians believed that because they would be notified that a request was modified by their patient, this information could actually be beneficial to have. For example: "I just want some notification that tells me [about the omission]...then I know to, in the next session, say, 'Hey, I saw that you chose not to tell me about mood. Let's talk about that"' (Clinician 7). If the application is able to make explicit an otherwise hidden process (the act of hiding information), this facilitates communication of a new type of potentially relevant information. The additional benefit of communicating this information, however, remains to be seen. One clinician noted that this may only serve to initiate conflict.

Despite the concerns, clinicians did recognize the benefits to the patient, that they are "the ultimate arbiter of what data of theirs is being shared" (Clinician 2). This was particularly useful when considering how data would be shared with multiple types of social support system roles (clinicians, parents, friends, etc.): "If you're in their support system... you're going to want more data points. Whereas a provider is going to be interested more in bigger-picture trends in order to inform medication management and treatment options." -Clinician 17

Overall, comments about data privacy highlight a potential tension between a clinician's desire to have access to all data collected about their patient, and the individual managing bipolar's desire to have more fine-grained control over data access. Some of these clinical concerns may be mitigated through the following additional design ideas elicited during the interviews:

- Alert clinicians when the patient chooses not to share a particular data item with them (and what that item was).

- Allow clinicians to automatically request recent data from patients before their appointment.

- Let individuals comment on the modifications they made to the request, including a reason why.

- Provide clear visualizations about which days you have data for, and which have been requested but not fulfilled.

5.2.5 Design 5: Data Commenting. 68\% of stoplight ratings on data commenting design features were green. Clinicians saw a variety of potential benefits from the data commenting feature, which 
would vary depending on the type of clinical practice. Particularly, commenting enabled patients to provide qualitative context to otherwise-quantitative data. Clinicians noted how data commenting would allow individuals to get support in a way that was tied directly to their tracked data, and gain the perspective of support system members.

\section{"It's the overlay [text comments] that often helps make sense of what the number means... I like the idea of being able to...observe trends in someone who maybe isn't noticing it or being able to draw someone's attention to $i t$, but also for someone who is noticing the trend and is the one making the ratings to be able to provide some additional context. I think this is great." -Clinician 2}

The data commenting feature also provides individuals with a method for creating a data-driven journal that may help in "challenging cognitive distortions" and lead to more day-to-day stability. In this sense, individuals may benefit by leaving a qualitative record of positive times in their life, that they can refer to during times of low mood: "Something that I've noticed is useful for a lot of people with bipolar disorder is like reminders to themselves from past states of mood... a comment that they made a month ago that said like 'felt really happy today' that might help them say like, 'oh, wow. I actually do feel happy sometimes"”-Clinician 7

Despite these noted benefits, clinicians had minor concerns about comments from social support system members (29\% yellow, $3 \%$ red). Clinicians thought that comments from support system members may often be unwanted by patients with bipolar disorder, and suggested that there be a feature for patients to disable commenting for particular variables or individuals: "I think it's a bit of a twoedged sword because I know a lot of patients who get annoyed with their family making comments like that... Mom puts in the comment 'are you taking your meds?' that's the kind of thing that just blows patients apart." -Clinician 13

Some clinicians mentioned they would not have the time to comment on data using the application. One suggestion was to create a drop-down list of common responses to speed up and/or standardize commenting. Another concern was the creation of a communication trail outside of traditional electronic health record (EHR) systems, suggesting a benefit to integrating a self-tracking application with existing EHR systems: "If somebody is in weekly therapy, a therapist would benefit from this and would be able to write back day-to-day probably. If they're seeing a psychiatrist once every six weeks for their medications. I don't know that they would be monitoring the daily input, but the ability to comment is probably helpful." -Clinician 9

This last quote highlights variability between clinicians. Some would be more willing to use this feature based on their own therapeutic style, while others may find this feature to be irrelevant. There was a single red rating, due to the potential for the data commenting to create a cluttered and overwhelming visual interface for the patient.

During interviews, the following additional design modifications were suggested by clinicians:

- Provide patients the ability to create a pre-session note, to plan for discussing certain topics.
- Include photos of the commenters alongside the data comments to provide a sense of familiarity.

- Allow for individuals to add comments via audio recording.

- Utilize icons and other symbols to mark specific data points, such as marking particular days with a star.

- Enable data commenting on photo uploads.

- Enable threaded replies on data comments.

- Enable individuals to comment on an absence of data.

5.2.6 Design 6: Custom Variables. Clinicians cited custom variables as a necessary feature of a flexible self-tracking app (56\% green), but shared a variety of caveats that might lead to reduced effectiveness in a clinical setting ( $38 \%$ yellow, $6 \%$ red). Clinicians were enthusiastic that the customization could lead to increased patient engagement and adoption of tracking behavior, but also collectively felt that it might be more challenging overall for the clinician to manage different ways in which different individuals customize their tracking.

"The advantage of having it this customizable is the patient might be more willing to do the tracking and that would be helpful for them, which is a good thing... as communicating what that means to a clinician, the more objective scales are probably easier for us to see quickly and get an overall understanding. [For] the more idiosyncratic scales, you'd have to have more of a discussion with the patient, but it does give you important information if they're willing to discuss it." -Clinician 9

Clinicians noted that the photo upload feature might be particularly relevant for children and adolescents, to help them engage in tracking. Others stressed the importance of having a quantitative rating tied to the photo uploads to assist in clinical interpretation. A common theme was the necessity for patients and clinicians to discuss the scales and come to an agreement about the tracking practices before use: "We've let them build their own rating scales, but they communicate them with us in advance so we know that if they're showing us a picture of a volcano, then we know that means they're really angry." -Clinician 9

The interviews highlighted the benefits of flexibility for the patients in creating their own scales, but also the necessity of coordination between the patient and clinician when doing so, such that the scales can be interpreted by the clinician and useful in practice. The two red ratings for this design were because clinicians did not think the photo uploads would be useful clinical information.

During interviews, the following additional design modifications were suggested by clinicians:

- Implement standard psychological instruments in the application, such as the Beck Depression Inventory (BDI), PatientHealth Questionnaire 9 (PHQ-9), Young Mania Rating Scale (YMRS), Lawton's Instrumental Activities of Daily Living (IADL), and others.

- Connect photo uploads with a corresponding numerical value, so clinicians can use numeric data with all patients.

5.2.7 Design 7: Dynamic Tracking Methods. 77\% of stoplight ratings for this design were green. Clinicians thought that patients would appreciate the ability to track certain variables automatically and believed that the more objective data would be therapeutically 
beneficial. In addition, multiple methods of tracking the same variable (automated and manual) would provide two perspectives on the same type of data, which could be useful in a clinical setting: "Some kids will bring me sleep records based on their FitBit and those are very useful... they're usually more accurate than what the patient can report so if you can do that [incorporate automated tracking] I think that would be a helpful... if they were doing both at the same time, you could see how they correlate." -Clinician 9

While only certain variables could be automatically tracked (such as sleep and activity), clinicians felt this feature would help patients be more willing to adopt tracking by reducing the overall tracking burden: "Frankly, I think that this is a great opportunity to be able to find other ways to measure so that it doesn't require as much deliberate tracking and logging. As easy as we can make it, the better." -Clinician 17

The primary concerns (12\% yellow) about this feature dealt with privacy and security-for example, ensuring that the data collected via smartphone sensors and peripherals was securely stored. In addition, there was one red rating due to the interface providing more information than was necessary.

During interviews, the following additional design modification was suggested by clinicians:

- Connect the app to online shopping platforms to monitor spending, which may indicate changes in mood state.

5.2.8 Design 8: Co-Tracking. Clinician feedback on co-tracking was generally positive, with $85 \%$ green responses. The largest perceived benefit in a clinical setting came from getting a diversity of perspectives about the patient's state. This is particularly important when patients are in states of mania and have potentially less self-awareness: "I like the idea of it, be a family member or friend who's also seeing someone outside of the treatment context and maybe getting more of a naturalistic sense of who they are and kind of how things are changing and ebbing and flowing in kind of, you know, their real world contexts... this is a great idea." -Clinician 2

Clinicians noted this particular feature would be relevant to children and adolescents who have their parents more heavily involved in their treatment. However, despite the benefits, clinicians noted that the co-tracking feature could introduce a new interface for conflict in relationships with social support system members $(12 \%$ yellow, 3\% red). There is a risk that patients may not feel a sense of independence in their tracking, and may not take control of their treatment plan: "Is it a self-management tool? Is it a mom management tool? ... Everyone's trying to be so supportive that it gets a bit codependent... I think there's a lot to this idea of letting someone else manage your mood or track your mood." -Clinician 6

While clinicians did perceive potential conflicts that may emerge in the co-tracking environment, overall, it was seen as a beneficial design feature that would provide useful multiple perspectives on treatment.

During interviews, the following additional design modification was suggested by a clinician:

- Enable individuals to store and share contact information of co-trackers, allowing these individuals to be reached in the case of significant changes.
5.2.9 Design 9: Action Plans. While clinicians were generally in favor of having infrastructure for action plans within the app (61\% green), they noted a variety of concerns dealing with liability and altering the patient-clinician relationship (36\% yellow, 3\% red). Clinicians appreciated the ability for the action plans to be readily available on the mobile phone, and be integrated in with existing data streams: "I love that. So they then have the opportunity to really tailor this action plan to their own desires and patterns to determine at what point things are triggered." -Clinician 17

Clinicians noted that action plans do not necessarily need to involve the clinician, and that notifications sent to social support system members may be more effective than contacting a provider immediately. Social support members could even serve as an intermediary between the clinician and patient. In addition, clinicians suggested that the app should include common resources such as a suicide hotline number or links to online bipolar support groups.

Clinicians were primarily concerned both about liability and the potential of creating unrealistic expectations between the patient and the clinician-imagine an action plan automatically triggered at $3 \mathrm{AM}$ by a patient in need of help. Many clinicians (understandably) expressed unwillingness to shoulder the burden of the expectations created by this scenario. These concerns might be mediated through discussions between the clinician and patient, as they work together to set up the action plans within the app.

During interviews, the following additional design modifications were suggested by clinicians:

- Allow for decision tree-style action plans, where multiple conditions must be met for a plan to be triggered.

- Allow for clinicians to opt-out of being part of action plans, to reduce liability.

- Create a repository of common action plans to serve as templates for individual patients, in order to reduce the burden of creating all new action plans.

- Support psychiatric advance directives.

\subsection{Additional Design Suggestions}

In this section, we present a list of design modifications or new design suggestions that arose during the interviews.

- Add the ability to create "homework" for patients in the app, such as daily or weekly checklists that help remind patients and break down the "homework" into smaller pieces.

- Ensure that the branding and naming of the application is discreet and innocuous to preserve patient privacy (i.e., the presence of the application on a phone should not tip off an onlooker that an individual is managing BD).

- Use the microphone to measure how quickly the patient is talking to identify the start of a hypomanic or manic mood state (for example, using methods from [46] or those reviewed in [57]).

- Develop a scoring system to encourage tracking and "gamify" the system to encourage consistent use (e.g., after [75]).

- Create a record of when clinicians or support system members view and interact with the data and display this information to patients so they know who is monitoring the data. 
- Prompt the individual to record data at random times throughout the day to mitigate the effect of framing and biases that may occur if data is entered at the same time of day.

- Frame prompts for certain variables (e.g., mood, irritability, suicidality) by asking patients to focus on symptom severity, rather than a simple temporal aggregation, which may mask rapid cycling throughout a day.

In summary, clinicians provided a diverse set of perspectives on the designs. The most pressing concerns, leading to multiple red ratings, were around incomplete data (4/13 red ratings), clinician workload (2/13), app complexity (2/13), and a concern that the data would not be useful (2/13). The other three red ratings were due to the potential for the organic imagery to be triggering to the patient, co-tracking promoting dependency in condition management, and liability issues with action plans using the application for emergency communication that would otherwise go through traditional medical channels. In our next section, we discuss a variety of design recommendations and guidelines based on the interviews, taking into account both the clinician feedback and the needs of the individuals with bipolar disorder.

\section{DISCUSSION-DESIGNING SELF-TRACKING SYSTEMS FOR WELL-BEING}

A variety of common themes arose during the inductive coding of the interview transcripts that expanded our view of the design space beyond our initial three design challenges. After iteratively grouping the codes, and limiting to codes from multiple clinicians and multiple design features, additional considerations emerged that might guide the design of effective self-tracking systems for well-being. In this section, we explore these considerations and relate them to the prior literature in self-tracking systems.

\subsection{Clinician Workload and Expectations in the Clinical Workflow}

A commonly cited concern was that of extra effort required by clinicians to interface with patients using self-tracking applications. Many of the interactions facilitated in the application represent new ways of interaction, such as a clinician commenting on a personal data stream, or sending requests to receive access to data. These additional activities were perceived by some clinicians as being prohibitively time consuming. While this concern has been noted in previous research in the context of clinicians using self-tracking data to make a physical health related diagnosis [77, 78], and as a general challenge of integrating patient-generated health data [67], ongoing monitoring of a patient's data stream represents an even larger workload burden.

More concerning than the workload considerations were the risks of creating additional inappropriate expectations for the clinicians revolving around the data. Clinicians were concerned that by having continuous access to patient data, they would become responsible for monitoring that data for concerning changes that may be indicative of a mental health crisis, such as a manic episode or a suicide attempt. If patients falsely believe that a clinician is monitoring a particular data stream, and that data changes for the worse, the patient may unrealistically expect the clinician to reach out or intervene in some way. In prior work [62], a patient participant described this as a "feeling of safety from knowing that others watch." While this may be feasible in some contexts, the clinicians we spoke to do not currently have an ongoing responsibility to monitor patient data in real time, and application design could unintentionally create this added burden or lead to unmet patient expectations [2]. To mitigate this, we suggest the application allow for explicit documentation and display of the agreed-upon role of the clinician with respect to the data. For example, a small information icon could be present next to data collection screens or data visualizations, reminding the patient when their provider has not agreed to actively monitor their data stream(s). This feature can help to ensure that patients do not have unrealistic expectations of clinicians, and clinicians may be more comfortable being involved with an application that provides real-time access to patient data.

\subsection{Modifying Traditional Clinician-Patient Interactions}

For many clinicians, all interaction with patients happens through institutionally controlled interfaces: in-person appointments, institutional phone calls, or through an electronic health record (EHR) messaging system. These interfaces have been established and maintained by medical institutions, and there may be risk-mitigation in place. For example, a phone call from a patient in crises may be triaged through an initial operator, and perhaps redirected to the public emergency dispatch number (such as 911 in the United States). Clinicians were concerned about liability exposure by using new interfaces to connect with patients. This finding motivates integration with existing medical interfaces, through artefacts brought into in-person appointments, reports uploaded to existing EHR systems, or complete integration on a common digital ecosystem.

In addition, the proposed data-sharing interface between patients and clinicians represents one where the patient, rather than the clinician, has the power, which may well be due to the fact that these interface designs were initially inspired by data-sharing practices reported by the individuals managing bipolar disorder and their social support system members [60]. Clinicians noted that they thought patients should be the final arbiter of their data sharing, but also that they wanted access to the complete dataset. In traditional medical settings, the doctor generally makes the decisions. In selftracking mHealth applications (like the one presented here), the patient makes the decision about what kind of data the clinician can see. This made clinicians uncomfortable, with some saying that unless they had the entire dataset, they would not use the data at all. This is similar to the existing concept of data believability [77], except that the clinician hesitation arises from a potential lack of completeness rather than a lack of trust in the original source of the data. From the clinician's point of view, the incompleteness of a patient's data stream is a type of uncertainty. Application design can incorporate strategies from uncertainty visualization [65] to clearly indicate to clinicians where the data is missing, in order to prevent incorrect conclusions from being drawn. For example, a continuous line of a patient's mood can be blurred out for the days during which a patient chose not to share data, as opposed to a break in the line where a patient did not track. 
Individuals managing BD expressed strong concern over their ability to control and selectively disclose their data [60, 66]. This desire may, at times, conflict with a clinician's desire to see the individual's entire data history. A system could be designed to prioritize either the clinician or the individual (by granting clinicians or therapists all data permissions by default, for example). However, data is collected with the audience in mind, and favoring the clinician may result in the individual changing how they collect data in the first place. As many of the variables important to managing bipolar disorder rely on subjective introspection (e.g., mood, irritability, suicidal ideation), they are particularly susceptible to so-called reactivity effects $[18,56]$.

In fact, the very negotiation of the data sharing process may itself be clinically valuable. Multiple clinicians brought up the potential for data-sharing changes to indicate either changes in mood state or as grounding for fruitful discussion with patients (e.g., Why didn't you want to share Monday's mood with me?). Whether this leads to conflict or harm perhaps depends on the individuals involved in the interface. As we'll see next, a diversity in desired use cases for the application suggests that application flexibility may be a prioritized design direction.

\subsection{Clinician Variability Necessitates Application Flexibility}

Enthusiasm for various features of the proposed system varied between clinicians, and this variation appeared to be connected to differences in clinical practice. The clinicians in our study saw differing numbers of patients per week, ranging from 2 to 40 . Accordingly, clinicians seeing more patients per week more commonly cautioned against possible workload concerns than clinicians seeing fewer patients. Additionally, clinicians varied in their eagerness to adopt a new interface for communication with their patients.

This variation in clinical applicability prompts reflection on the different ways that a clinician might engage with the proposed system. To summarize key takeaways from our interviews, we present two different vignettes of hypothetical clinicians using a potential self-tracking app in different ways, grounded in the responses provided by clinical participants with varying backgrounds. These vignettes were constructed based on common themes emerging from clinician interviews, and the personas were chosen to distinguish between clinicians whose practices differ based on number of patients, whether situated in a public vs. private setting, and the types of mental health services provided. The first is of John, a clinical psychologist running a private practice specializing in mood disorders, with relatively few clients. The second is of Jenn, a psychiatrist working at a large public hospital, who sees many clients each week.

6.3.1 Vignette One - Small, Private Practice Psychology. John runs his own private practice with a small office where he sees 15 patients per week. Most of his patients are relatively well-off, and pay for service largely through private insurance. Fohn focuses on mood disorders, and about half of his patients have been diagnosed with bipolar disorder. Fohn sees each of his patients once a week for about an hour for psychotherapy. Many of his patients also see a psychiatrist around once per month. Although fohn does coordinate with his patients' psychiatrists by sharing clinical notes, he is generally not involved in the prescription of psychiatric medication.

fohn started recommending the use of a self-tracking application as part of his therapeutic practice. When seeing a client diagnosed with bipolar disorder for the first time, fohn provides an overview of how to incorporate IPRST [24] into daily living, including the use of a selftracking application. Fohn provides an overview of the application and works with the client to configure the app to suit their particular needs. fohn recommends that individuals share data continuously with him as a clinician, which makes it easy for him to review patient data before appointments, and to identify if and when particular issues arise between appointments.

While setting up the application, fohn again makes it clear that the application is not intended to diagnose or respond to crises, but rather helps to facilitate adherence to regular daily rhythms. fohn makes an effort to comment on each patient's data stream between appointments, providing encouragement and support. Fohn uses the data to better understand each client's daily lived experience, and particularly values seeing the multiple perspectives provided by co-tracking. Fohn is willing to set up automated action plans that involve notifications on his phone, but has made sure to include additional measures in the plan in the case he is unavailable or unable to respond in time, such as including the suicide hotline number and the phone number of a local psychiatric hospital.

6.3.2 Vignette Two: Psychiatry in a Large Public Hospital. Fenn is a medical doctor with a specialty in psychiatry. She works at a large public hospital in a major metropolitan area, and has over 150 patients under her care, the vast majority of whom have bipolar disorder. She sees each patient about once every six weeks, and sees around 30 patients a week in total. Appointments for repeat patients are 30 minutes and usually consist of a clinical evaluation grounded in standard psychometric instruments, used primarily to inform medication changes.

fenn recommends that her patients use a self-tracking app to help them manage their own condition and as an input to her clinical decision making around medication selection and dosage. For new patients, she takes the time to set up the application with them, explain the features, and prepare a variety of action plans. However, fenn herself does not use the application, and is not directly connected to the patient's data stream or automated action plans. Instead, Jenn requests that the patient export data before an appointment, and either upload the report to the hospital's EHR system, or simply show the data on the phone during an appointment as an inclusion artifact [12, 53]. In doing so, Jenn avoids the clinical and legal issues of using a separate patient interface, as data is communicated only using existing interaction and data-sharing mechanisms (e.g., appointments, EHR-based messaging).

6.3.3 Comparing Vignettes. The examples above highlight how a system can be flexible enough to accommodate different use cases by individuals with the same high-level "role"-that of a clinician. We saw overlap in clinical practice, as both hypothetical clinicians took the time to educate their patient how to use the app effectively, set it up with them, and clarify expectations about what role the app would play in clinical practice, as well as the extent to which each clinician might interact with the patient through the app. However, clinician use diverged significantly after the initial set-up appointment across these two vignettes. The first vignette 
highlights how some clinicians may choose to be more involved with their patients' data on an on-going basis, using the application as a new interface with the patient. The second vignette, on the other hand, highlights the use of the application as an augmentation of an existing patient-clinician interface: the clinical appointment.

Our findings highlight the necessity of accommodating variability in clinical approach when designing systems for well-being. Individuals with SMI may seek treatment from multiple types of providers (for example, both a therapist and psychiatrist), and these different clinical roles may use the application in different ways. Some clinicians may not wish to be involved in self-tracking applications at all, motivating a design that "works" even without direct clinician use.

\subsection{The Multiplicative Patient: Intra-Individual Roles, Conflict, and Communication}

Clinicians highlighted concerns that would only be valid when an individual is in a particular affective state. It became clear that when individuals with bipolar enter a state of mania, for example, their behavior changes so significantly as to warrant different design affordances. This means that the primary user for which this system is intended-the individual with bipolar disorder-may actually be best represented by multiple roles within the designed system. The system must accommodate the "(hypo)manic self," "depressed self," and "euthymic self," and, perhaps, a "rapid-cycling self”. These intra-individual roles, applicable based on the current cognitive state of the individual, have different design considerations not only in feature selection, but also in interface design. The case of bipolar disorder motivates an understanding of the behavioral tendencies of individuals in each role, and design considerations may not be evenly spread across temporal distributions. For example, individuals with bipolar disorder are in a manic state no more than $1 \%$ of the time [40], but an application may need to have significant affordances to accommodate the state of mania.

6.4.1 Designing for Intra-Individual Roles. Individuals in a state of mania are often, in the eyes of their clinicians, unpredictable. They may be unlikely to perform consistent self-tracking, and may possibly take surprising actions like removing everyone from the application's data-sharing access list. However, the same individual in a state of euthymia or depression may not do this. The potential for intra-individual conflict $[1,31]$ creates a diversity of requirements in the design of a system for supporting well-being of the individual. In systems supporting mental health, intra-individual conflict can occur when the behavior of an individual in one state negatively affects the needs of the same individual in a different state. In the case where a manic individual deletes everyone from the app, this behavior of the manic self harms the ability of the euthymic self to maintain its state of euthymia (by damaging social relations, for example, or hiding necessary data from their clinician).

Clinicians also highlighted the potential to utilize the diversity of mood states in a therapeutically beneficial way. The datacommenting feature (design five) provides affordances not only for supporting communication between individuals, but also for asynchronous intra-individual communication. When a stable, euthymic individual leaves a motivating text comment for the future, depressed self, the system is accommodating a type of intra-individual communication that would otherwise not be possible, because the individual is only in a single state at a single point in time. This, we propose, as a key feature for self-tracking systems that support well-being: the ability for individuals to communicate across time with their various selves in different states of well-being.

To summarize our findings on intra-individual roles, we present a network diagram highlighting potential conflict and potential therapeutic communication within a single individual across different affective states. Solid blue arrows indicate ideal roles for application setup, the finely dashed red arrow indicates potential for intra-individual conflict, and coarsely dashed green arrows indicate the potential for intra-individual therapeutic benefit.

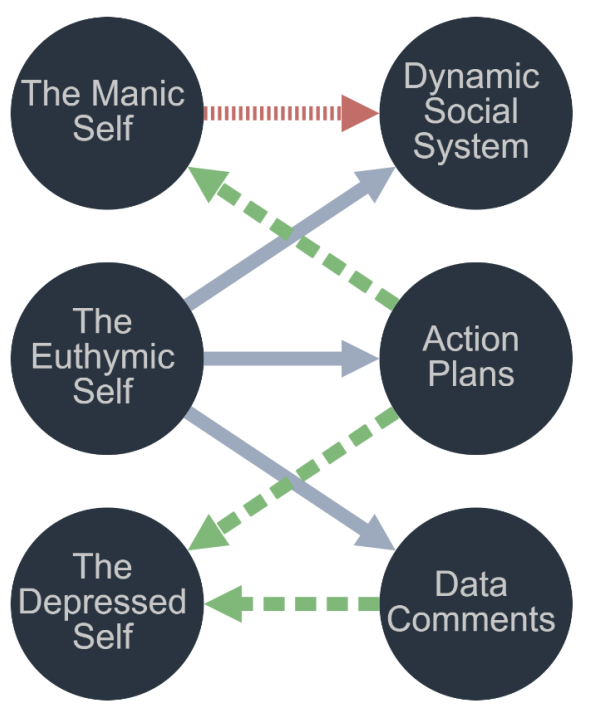

Figure 4: A network diagram showing relationships between intra-individual roles and well-being application features, informed by clinicians' responses to the proposed interface designs and their novel feature recommendations. Solid blue arrows indicate ideal roles for application setup, the finely dashed red arrow indicates potential for intraindividual conflict, and coarsely dashed green arrows indicate the potential for intra-individual therapeutic benefit.

As seen in Figure 4, the euthymic self is best suited to configure the application and manage data-sharing permissions for a dynamic social system, while the manic self may potentially cause harm by removing support members from the app. In action plans, the euthymic self "communicates" a plan of action to future selves (often, in collaboration with their clinician), which is largely expected to benefit the individual in states both of mania and depression. Euthymic individuals also have the opportunity to communicate to future depressed selves through data commenting-for example, by creating annotations on self-tracked data from a time when the lived experience was generally positive.

Understanding the diversity of states within a single user motivates design affordances to mitigate conflict and promote therapeutic communication. For example, to mitigate potential harm resulting from a manic individual removing all contacts from the 
app, the application could allow for "saving" a social system configuration. A euthymic individual can create a "save state" that stores the data-sharing permissions for each member of their social system. If, after a manic episode, they find all their contacts have been removed by their manic self, then the individual can "restore" their social system to a previous state. While this design feature may be helpful for preserving the usefulness of the application, it could also serve to reify one's experience of "restarting" after a manic episode, and improve psychosocial factors that contribute to recovery [35] The application could send messages or educational material to those in the individual's support system, with recommendations on how to best support an individual after a manic episode.

While the stark differences in mood state in BD motivate designing specifically for intra-individual roles, these considerations apply more generally to systems for supporting well-being. Significant changes in mental state are common to a variety of SMI, and to life in general. By considering the different cognitive and affective states that an individual may be in, systems can better support the diversity of user needs within a single individual.

In addition, an individual's changing sense of identity is a significant factor in behavior change outcomes [38] or overcoming the shock of an SMI diagnosis [49]. In the case of smoking cessation, for example, an individual may struggle in shifting identity from being a smoker to a non-smoker [19]. This "identity revision" is an important aspect of solidifying behavior change [38]. Systems that explicitly affirm the existence of multiple (changing) components of the individual's sense of self (e.g., through multiple roles for a single user) may be more effective than a system that represents the individual as having a single, unified role. Supporting distinct identities within an application may support the revision of one's identity in a desired direction.

\subsection{Effects On and From Social Systems}

While our interviews were largely focused on the patient-clinician relationship, clinicians often provided insight into how a supporting social system can influence and be influenced by a system for managing well-being.

Reactivity effects are a known effect in personal informatics systems [56]. Reactivity is when the act of measuring a variable leads to change in the variable itself (or some other variable). In our proposed system, clinicians helped us identify a type of social reactivity, one that may occur when a mental prediction of the effect of communicating a piece of data modifies the very collection of the data. This has been noted elsewhere, in the general sense, as the Hawthorne effect [52]. In the case of continuous data sharing, the individual collecting data knows, at the time of data collection, who will have immediate access to this particular set of data. The individual may modify how they collect data based on some prediction of how sharing that data may influence a relationship. Perhaps a child does not want to share a variable "fights with my brother" (a hypothetical custom variable mentioned by clinician 13) for fear of punishment from a parent. If the system is set up to automatically provide this data to the parent, then the child may think twice about honest reporting of each (potentially relevant) encounter.

Sharing data via "discrete requests" (design four) provides one potential mitigation of reactivity effects. By decoupling the process of sharing data from the process of collecting data, we expect the social reactivity effects to be lessened, since the individual can choose to never share specific data after it has been collected. However, the requesting and sharing process is likely a new type of interaction between an individual and their support system members. Clinicians noted that this may be a new source of conflict within a social system, such as if a support member gets upset that a particular data request is modified or denied.

Providing social system members an ability to communicate in the same context as the self-tracked data (via data-commenting, design 5) evoked a few clinical concerns and calls for careful design consideration. Some clinicians mentioned that individuals may get annoyed if a close relation comments on particular data, when they may not want to open up a digital conversation about that data or direct attention towards a particular variable. The commenting, in this case, could actually provoke new conflict or tension in a social system. On the other hand, the individual may feel ignored if nobody comments on their data, especially in the case of data indicating a trend away from a stable or euthymic state. This resonates with previous studies highlighting the importance of "authenticating one's experience" by having a receptive audience [66]. Clinicians suggested two design considerations to help mitigate these risks: (1) providing fine-grained control of data commenting permissions, and (2) displaying information about who has "seen" particular data entries. The nuances of how the data-commenting feature is designed, and its use within the patient's dynamic social system [60], will moderate the extent to which data-contextualized communication is harmful or helpful to the individual.

The notion of relational recovery [69] prompts the inclusion of social interfaces within systems for well-being, such as data sharing and data commenting. However, these interfaces and associated social reactivity effects create the potential for unintended consequences, such as influencing the actual data collection itself or creating new types of conflict in existing social systems. Effective design can support beneficial social interactions around self-tracked data, while limiting the risks of creating new social communication channels.

\subsection{Statement of Limitations}

We limited our study to include clinicians local to the Denver metro area in the United States. The extent to which the clinical practices of these individuals represent the general clinical approach to treating bipolar disorder worldwide is unknown. In addition, the practice of self-tracking may not be an efficacious treatment for all patients, potentially adding an unwanted burden [47]. In addition, the perspectives presented in this work are solely those of clinicians. While clinicians may have accurate perceptions about their clients' needs and behavior, we refer to prior work [49, 50, 60, 74] as references for direct design feedback from those individuals actively managing bipolar disorder of their own. Additionally, while the clinicians surveyed had a variety of diverse backgrounds (see Table 1), our sample size of 17 from a single locality may limit generalizability.

Lastly, the interface wireframes presented in this study were not fully functional. Interacting with an actual functioning application may result in clinicians providing different feedback. 


\section{CONCLUSION AND FUTURE WORK}

In this study, we interviewed a diverse set of mental health clinicians to elicit a clinical perspective on nine novel features for a self-tracking application to support the management of bipolar disorder. In doing so, we discovered unique design constraints and opportunities applicable to applications for supporting BD, as well as systems for supporting well-being, in general.

Clinical integration of patient generated mental health data is hindered by similar concerns clinicians have about physical health data, such as added workload and data believability. However, as changes in mental health data may be indicative of an impending crisis (such as a suicide attempt), real-time monitoring of this data is a large burden that clinicians are fearful to adopt. This reluctance motivates the creation of a patient-controlled system for real-time monitoring of mental health data, with appropriate and timely intervention.

Part of the solution may be automated, data-driven action plans (design 9), such as notifying trusted contacts when an individual records large deviations in self-reported mood values or begins logging instances of suicidal ideation. However, we discovered a type of social reactivity that can occur when self-tracked data is continuously shared with others (design 3)-the individual's awareness of who will see their data impacts how they self-track (choosing not to track, or providing "false" information). Applications may be able to mitigate this by decoupling data collection from data sharing, relying on discrete "requests" and "shares" of self-tracked data that occur after the data has been recorded (design 4).

Another, perhaps more robust solution is to gather multiple perspectives on the individual's mental state by allowing trusted contacts to enter data on behalf of, and about, the individual (design 8). While clinicians believed this would provide a valuable second perspective in a clinical context, having one person co-track for another, especially for subjective variables such as mood or irritability, is a largely unstudied process. The complex emotional system formed by two individuals in a close partnership is full of feedback loops [10], and the effects of introducing a co-tracking intervention are unknown. A fruitful direction for research is learning how to design a system that leads to an improved capacity for a partnership to moderate negative emotional states, perhaps by improving empathic accuracy via mutual co-tracking of emotions.

The stark contrast between cognitive states of an individual managing bipolar disorder highlights the multiplicity of selves within a single individual. Conceptualizing a person as multiple intra-individual selves all accessing the same application offers opportunities for design to facilitate cooperation between selves across time (such as with data commenting, design 5, and action plans, design 9). The HCI community may benefit from further research on how to best design systems that support multiple intraindividual roles for a single person. While researchers have made effort towards "authenticating" affect-based roles through emotion recognition (see [37] for a review), much remains to be explored about using these roles to reify and support different components of an individual's dynamic identity, especially for those managing a serious mental illness such as bipolar disorder.

\section{ACKNOWLEDGMENTS}

We thank the participating clinicians for their helpful feedback and insights. In addition, we would like to thank Jang-hee Cho and Elizabeth Murname for their constructive comments on the manuscript. This research was supported by a seed grant from the Renée Crown Wellness Institute at the University of Colorado Boulder.

\section{REFERENCES}

[1] Catherine E. Amiot, Winnifred R. Louis, Sarah Bourdeau, and Oulma Maalouf. 2017. Can harmful intergroup behaviors truly represent the self?: The impact of harmful and prosocial normative behaviors on intra-individual conflict and compartmentalization. Self and Identity 16, 6 (Nov. 2017), 703-731. https: //doi.org/10.1080/15298868.2017.1305442

[2] Tariq Osman Andersen, Jørgen Peter Bansler, Finn Kensing, Jonas Moll, Troels Mønsted, Karen Dam Nielsen, Olav Wendelboe Nielsen, Helen Høgh Petersen, and Jesper Hastrup Svendsen. 2019. Aligning concerns in telecare: Three concepts to guide the design of patient-centred e-health. Computer Supported Cooperative Work (CSCW) 28, 6 (Oct. 2019), 1039-1072. https://doi.org/10.1007/s10606-0189309-1

[3] Ross J. Baldessarini and Leonardo Tondo. 2003. Suicide risk and treatments for patients with bipolar disorder. Fournal of the American Medical Association 290, 11 (Sept. 2003), 1517-1519. https://doi.org/10.1001/jama.290.11.1517

[4] Jakob E. Bardram, Mads Frost, Károly Szántó, Maria Faurholt-Jepsen, Maj Vinberg, and Lars Vedel Kessing. 2013. Designing mobile health technology for bipolar disorder: A field trial of the MONARCA system. In Proceedings of the SIGCHI Conference on Human Factors in Computing Systems (CHI '13). ACM Press, New York, NY, 2627-2636. https://doi.org/10.1145/2470654.2481364

[5] Jakob E. Bardram, Mads Frost, Károly Szántó, and Gabriela Marcu. 2012. The MONARCA self-assessment system: A persuasive personal monitoring system for bipolar patients. In Proceedings of the 2nd ACM SIGHIT International Health Informatics Symposium (IHI '12). ACM Press, New York, NY, 21-30. https://doi. org $/ 10.1145 / 2110363.2110370$

[6] Michael Bauer, Paul Grof, Laszlo Gyulai, Natalie Rasgon, Tasha Glenn, and Peter C. Whybrow. 2004. Using technology to improve longitudinal studies: Self-reporting with ChronoRecord in bipolar disorder. Bipolar Disorders 6, 1 (2004), 67-74. https://doi.org/10.1046/j.1399-5618.2003.00085.x

[7] Amit Baumel, Frederick Muench, Stav Edan, and John M. Kane. 2019. Objective user engagement with mental health apps: Systematic search and panel-based usage analysis. Journal of Medical Internet Research 21, 9 (2019), e14567. https: //doi.org/10.2196/14567

[8] Linda Birt, Suzanne Scott, Debbie Cavers, Christine Campbell, and Fiona Walter. 2016. Member checking: A tool to enhance trustworthiness or merely a nod to validation? Qualitative Health Research 26, 13 (Nov. 2016), 1802-1811. https: //doi.org/10.1177/1049732316654870

[9] Jedediah M. Bopp, David J. Miklowitz, Guy M. Goodwin, Will Stevens, Jennifer M. Rendell, and John R. Geddes. 2010. The longitudinal course of bipolar disorder as revealed through weekly text messaging: A feasibility study. Bipolar Disorders 12, 3 (2010), 327-334. https://doi.org/10.1111/j.1399-5618.2010.00807.x

[10] Emily A. Butler. 2017. Emotions are temporal interpersonal systems. Current Opinion in Psychology 17 (Oct. 2017), 129-134. https://doi.org/10.1016/j.copsyc. 2017.07.005

[11] Steven Chan, John Torous, Ladson Hinton, and Peter Yellowlees. 2015. Towards a framework for evaluating mobile mental health apps. Telemedicine and e-Health 21, 12 (July 2015), 1038-1041. https://doi.org/10.1089/tmj.2015.0002

[12] Chia-Fang Chung, Kristin Dew, Allison Cole, Jasmine Zia, James Fogarty, Julie A. Kientz, and Sean A. Munson. 2016. Boundary negotiating artifacts in personal informatics: Patient-provider collaboration with patient-enerated data. In Proceedings of the 19th ACM Conference on Computer-Supported Cooperative Work \& Social Computing (CSCW '16). ACM Press, New York, NY, 770-786. https://doi.org/10.1145/2818048.2819926

[13] Margaret S. Clark, Katherine R. Von Culin, Elizabeth Clark-Polner, and Edward P. Lemay, Jr. 2017. Accuracy and projection in perceptions of partners' recent emotional experiences: Both minds matter. Emotion 17, 2 (March 2017), 196-207. https://doi.org/10.1037/emo0000173

[14] Western Electric Company. 1956. Statistical Quality Control Handbook. Western Electric Company, Easton, PA.

[15] Juliet Corbin and Anselm Strauss. 2014. Basics of Qualitative Research: Techniques and Procedures for Developing Grounded Theory (4th ed.). SAGE Publications, Los Angeles, CA.

[16] Gavin Doherty, David Coyle, and Mark Matthews. 2010. Design and evaluation guidelines for mental health technologies. Interacting with Computers 22, 4 (July 2010), 243-252. https://doi.org/10.1016/j.intcom.2010.02.006

[17] Daniel A. Epstein, An Ping, James Fogarty, and Sean A. Munson. 2015. A lived informatics model of personal informatics. In Proceedings of the 2015 ACM International Joint Conference on Pervasive and Ubiquitous Computing (UbiComp '15). ACM Press, New York, NY, 731-742. https://doi.org/10.1145/2750858.2804250 
[18] Robert Epstein. 1996. Self Help Without the Hype. Performance Management Publications, Tucker, GA.

[19] Juan Manuel Falomir and Federica Invernizzi. 1999. The role of social influence and smoker identity in resistance to smoking cessation. Swiss fournal of Psychology / Schweizerische Zeitschrift für Psychologie / Revue Suisse de Psychologie 58, 2 (1999), 73-84. https://doi.org/10.1024/1421-0185.58.2.73

[20] Jodi Forlizzi. 2018. Moving beyond user-centered design. Interactions 25, 5 (Aug. 2018), 22-23. https://doi.org/10.1145/3239558

[21] Ellen Frank. 2007. Interpersonal and social rhythm therapy: A means of improv ing depression and preventing relapse in bipolar disorder. Fournal of Clinical Psychology 63, 5 (2007), 463-473. https://doi.org/10.1002/jclp.20371

[22] Ellen Frank, David J. Kupfer, Michael E. Thase, Alan G. Mallinger, Holly A. Swartz, Andrea M. Fagiolini, Victoria Grochocinski, Patricia Houck, John Scott, Wesley Thompson, and Timothy Monk. 2005. Two-year outcomes for interpersonal and social rhythm therapy in individuals with bipolar I disorder. Archives of General Psychiatry 62, 9 (Sept. 2005), 996-1004. https://doi.org/10.1001/archpsyc.62.9.996

[23] Ellen Frank, Holly A. Swartz, and Elaine Boland. 2007. Interpersonal and socia rhythm therapy: An intervention addressing rhythm dysregulation in bipolar disorder. Dialogues in Clinical Neuroscience 9, 3 (2007), 325-332. https://doi.org/ 10.31887/DCNS.2007.9.3/efrank

[24] Ellen Frank, Holly A. Swartz, and David J. Kupfer. 2000. Interpersonal and social rhythm therapy: Managing the chaos of bipolar disorder. Biological Psychiatry 48, 6 (Sept. 2000), 593-604. https://doi.org/10.1016/S0006-3223(00)00969-0

[25] Mads Frost, Afsaneh Doryab, Maria Faurholt-Jepsen, Lars Vedel Kessing, and Jakob E. Bardram. 2013. Supporting disease insight through data analysis: Refinements of the MONARCA self-assessment system. In Proceedings of the 2013 ACM International foint Conference on Pervasive and Ubiquitous Computing (UbiComp '13). ACM Press, New York, NY, 133-142. https://doi.org/10.1145/2493432.2493507

[26] Emma Gliddon, Steven J. Barnes, Greg Murray, and Erin E. Michalak. 2017. Online and mobile technologies for self-management in bipolar disorder: A systematic review. Psychiatric Rehabilitation fournal 40, 3 (Sept. 2017), 309-319. https: //doi.org/10.1037/prj0000270

[27] VERBI GmbH. 2019. MAXQDA 2020. https://www.maxqda.com

[28] Frederick K. Goodwin and Kay Redfield Jamison. 2007. Manic-Depressive Illness: Bipolar Disorders and Recurrent Depression. Vol. 2. Oxford University Press, Oxford, UK.

[29] Iria Grande, Michael Berk, Boris Birmaher, and Eduard Vieta. 2016. Bipolar disorder. The Lancet 387, 10027 (April 2016), 1561-1572. https://doi.org/10.1016/ S0140-6736(15)00241-X

[30] Agnes Grünerbl, Amir Muaremi, Venet Osmani, Gernot Bahle, Stefan Öhler, Gerhard Tröster, Oscar Mayora, Christian Haring, and Paul Lukowicz. 2015 Smartphone-based recognition of states and state changes in bipolar disorder patients. IEEE fournal of Biomedical and Health Informatics 19, 1 (Jan. 2015), 140-148. https://doi.org/10.1109/JBHI.2014.2343154

[31] Susan Harter. 1999. The Construction of the Self: A Developmental Perspective Guilford Press, New York, NY.

[32] Maree L. Inder, Marie T. Crowe, Stephanie Moor, Suzanne E. Luty, Janet D. Carter, and Peter R. Joyce. 2008. "I actually don't know who I am": The impact of bipolar disorder on the development of self. Psychiatry: Interpersonal and Biological Processes 71, 2 (2008), 123-133. https://doi.org/10.1521/psyc.2008.71.2.123

[33] Thomas R. Insel. 2008. Assessing the economic costs of serious mental illness. American fournal of Psychiatry 165, 6 (June 2008), 663-665. https://doi.org/10. 1176/appi.ajp.2008.08030366

[34] Sachin H. Jain, Brian W. Powers, Jared B. Hawkins, and John S. Brownstein. 2015. The digital phenotype. Nature Biotechnology 33, 5 (May 2015), 462-463. https://doi.org/10.1038/nbt.3223

[35] Sheri L. Johnson and Ivan Miller. 1997. Negative life events and time to recovery from episodes of bipolar disorder. Fournal of Abnormal Psychology 106, 3 (Aug. 1997), 449-457. https://doi.org/10.1037/0021-843X.106.3.449

[36] David A. Kahn, Gary S. Sachs, David J. Printz, Daniel Carpenter, John P. Docherty, and Ruth Ross. 2000. Medication treatment of bipolar disorder 2000: A summary of the expert consensus guidelines. Fournal of Psychiatric Practice 6, 4 (2000), 197-211. https://doi.org/10.1097/00131746-200007000-00004

[37] Eiman Kanjo, Luluah Al-Husain, and Alan Chamberlain. 2015. Emotions in context: Examining pervasive affective sensing systems, applications, and analyses. Personal and Ubiquitous Computing 19, 7 (Oct. 2015), 1197-1212. https://doi.org/10.1007/s00779-015-0842-3

[38] Margaret H. Kearney and Joanne O'Sullivan. 2003. Identity shifts as turning points in health behavior change. Western fournal of Nursing Research 25, 2 (March 2003), 134-152. https://doi.org/10.1177/0193945902250032

[39] Gerald L. Klerman. 1984. Interpersonal Psychotherapy of Depression. Basic Books, New York, NY.

[40] Ralph W. Kupka, Lori L. Altshuler, Willem A. Nolen, Trisha Suppes, David A. Luckenbaugh, Gabriele S. Leverich, Mark A. Frye, Paul E. Keck, Susan L. McElroy, Heinz Grunze, and Robert M. Post. 2007. Three times more days depressed than manic or hypomanic in both bipolar I and bipolar II disorder1. Bipolar Disorders 9, 5 (2007), 531-535. https://doi.org/10.1111/j.1399-5618.2007.00467.x

[41] Sue Lauder, Andrea Chester, David Castle, Seetal Dodd, Lesley Berk, Britt Klein, David Austin, Monica Gilbert, James A. Chamberlain, Greg Murray, Carolynne
White, Leon Piterman, and Michael Berk. 2013. Development of an online intervention for bipolar disorder. www.moodswings.net.au. Psychology, Health \& Medicine 18, 2 (March 2013), 155-165. https://doi.org/10.1080/13548506.2012. 689840

[42] Ian Li, Anind Dey, and Jodi Forlizzi. 2010. A stage-based model of personal informatics systems. In Proceedings of the SIGCHI Conference on Human Factors in Computing Systems (New York, NY) (CHI '10). ACM Press, New York, NY, 557-566. https://doi.org/10.1145/1753326.1753409

[43] Daniel Z. Lieberman, Susan Swayze, and Frederick K. Goodwin. 2011. Best practices: An automated internet application to help patients with bipolar disorder track social rhythm stabilization. Psychiatric Services 62, 11 (Nov. 2011), 12671269. https://doi.org/10.1176/ps.62.11.pss6211_1267

[44] Yvonna S. Lincoln. 2007. Naturalistic Inquiry. In The Blackwell Encyclopedia of Sociology. Blackwell Publishers, Malden, MA. https://doi.org/10.1002/ 9781405165518.wbeosn006

[45] Ross J. Lordon, Sean P. Mikles, Laura Kneale, Heather L. Evans, Sean A. Munson, Uba Backonja, and William B. Lober. 2020. How patient-generated health data and patient-reported outcomes affect patient-clinician relationships: A systematic review. Health Informatics fournal 26, 4 (Dec. 2020), 2689-2706. https://doi.org/ $10.1177 / 1460458220928184$

[46] Hong Lu, Denise Frauendorfer, Mashfiqui Rabbi, Marianne Schmid Mast, Gokul T. Chittaranjan, Andrew T. Campbell, Daniel Gatica-Perez, and Tanzeem Choudhury. 2012. StressSense: Detecting stress in unconstrained acoustic environments using smartphones. In Proceedings of the 2012 ACM Conference on Ubiquitous Computing (UbiComp '12). ACM Press, New York, NY, 351-360. https://doi.org/10.1145/ 2370216.2370270

[47] Benjamin Marent, Flis Henwood, and Mary Darking. 2018. Ambivalence in digital health: Co-designing an mHealth platform for HIV care. Social Science \& Medicine 215 (Oct. 2018), 133-141. https://doi.org/10.1016/j.socscimed.2018.09.003

[48] Mark Matthews, Saeed Abdullah, Elizabeth Murnane, Stephen Voida, Tanzeem Choudhury, Geri Gay, and Ellen Frank. 2016. Development and evaluation of a smartphone-based measure of social rhythms for bipolar disorder. Assessment 23, 4 (2016), 472-483. https://doi.org/10.1177/1073191116656794

[49] Mark Matthews, Elizabeth Murnane, and Jaime Snyder. 2017. Quantifying the changeable self: The role of self-tracking in coming to terms with and managing bipolar disorder. Human-Computer Interaction 32, 5-6 (2017), 413-446. https: //doi.org/10.1080/07370024.2017.1294983

[50] Mark Matthews, Elizabeth Murnane, Jaime Snyder, Shion Guha, Pamara Chang, Gavin Doherty, and Geri Gay. 2017. The double-edged sword: A mixed methods study of the interplay between bipolar disorder and technology use. Computers in Human Behavior 75 (2017), 288-300. https://doi.org/10.1016/j.chb.2017.05.009

[51] Alexis M. May, Alexander O. Crenshaw, Feea Leifker, Craig J. Bryan, and Brian R.W. Baucom. 2019. Knowledge of suicide history, current depressive symptoms, and future suicide risk within couples. Behaviour Research and Therapy 120 (Sept. 2019), 103394. https://doi.org/10.1016/j.brat.2019.03.015

[52] Jim McCambridge, John Witton, and Diana R. Elbourne. 2014. Systematic review of the Hawthorne effect: New concepts are needed to study research participation effects. Fournal of Clinical Epidemiology 67, 3 (March 2014), 267-277. https: //doi.org/10.1016/j.jclinepi.2013.08.015

[53] Helena M. Mentis, Anita Komlodi, Katrina Schrader, Michael Phipps, Ann GruberBaldini, Karen Yarbrough, and Lisa Shulman. 2017. Crafting a view of self-tracking data in the clinical visit. In Proceedings of the 2017 CHI Conference on Human Factors in Computing Systems (CHI '17). ACM Press, New York, NY, 5800-5812. https://doi.org/10.1145/3025453.3025589

[54] Kathleen R. Merikangas, Robert Jin, Jian-Ping He, Ronald C. Kessler, Sing Lee, Nancy A. Sampson, Maria Carmen Viana, Laura Helena Andrade, Chiyi Hu, Elie G. Karam, Maria Ladea, Maria Elena Medina-Mora, Yutaka Ono, Jose PosadaVilla, Rajesh Sagar, J. Elisabeth Wells, and Zahari Zarkov. 2011. Prevalence and correlates of bipolar spectrum disorder in the World Mental Health Survey Initiative. Archives of General Psychiatry 68, 3 (March 2011), 241-251. https: //doi.org/10.1001/archgenpsychiatry.2011.12

[55] David J. Miklowitz, Michael W. Otto, Ellen Frank, Noreen A. Reilly-Harrington, Stephen R. Wisniewski, Jane N. Kogan, Andrew A. Nierenberg, Joseph R. Calabrese, Lauren B. Marangell, Laszlo Gyulai, Mako Araga, Jodi M. Gonzalez, Edwin R. Shirley, Michael E. Thase, and Gary S. Sachs. 2007. Psychosocial treatments for bipolar depression: A 1-year randomized trial from the systematic treatment enhancement program. FAMA Psychiatry 64, 4 (April 2007), 419-426. https://doi.org/10.1001/archpsyc.64.4.419

[56] Raymond G. Miltenberger. 2008. Behavior Modification: Principles and Procedures (4th ed.). Thomson Wadsworth, Belmont, CA.

[57] Scott Monteith, Tasha Glenn, John Geddes, Peter C. Whybrow, and Michael Bauer. 2016. Big data for bipolar disorder. International Journal of Bipolar Disorders 4, Article 10 (April 2016), 18 pages. https://doi.org/10.1186/s40345-016-0051-7

[58] Elizabeth L. Murnane, Dan Cosley, Pamara Chang, Shion Guha, Ellen Frank, Geri Gay, and Mark Matthews. 2016. Self-monitoring practices, attitudes, and needs of individuals with bipolar disorder: Implications for the design of technologies to manage mental health. Fournal of the American Medical Informatics Association 
23, 3 (2016), 477-484. https://doi.org/10.1093/jamia/ocv165

[59] Elizabeth L. Murnane, Jaime Snyder, Stephen Voida, Matthew J. Bietz, Mark Matthews, Sean Munson, and Laura R. Pina. 2018. Social issues in personal informatics: Design, data, and infrastructure. In Companion of the 2018 ACM Conference on Computer Supported Cooperative Work and Social Computing (CSCW '18). ACM, New York, 471-478. https://doi.org/10.1145/3272973.3273016

[60] Elizabeth L. Murnane, Tara G. Walker, Beck Tench, Stephen Voida, and Jaime Snyder. 2018. Personal informatics in interpersonal contexts: Towards the design of technology that supports the social ecologies of long-term mental health management. Proceedings of the ACM on Human-Computer Interaction 2, CSCW, Article 127 (Nov. 2018), 27 pages. https://doi.org/10.1145/3274396

[61] Jaime Muñoz-Arteaga, Ricardo Mendoza González, Miguel Vargas Martin, Jean Vanderdonckt, and Francisco Álvarez Rodríguez. 2009. A methodology for designing information security feedback based on User Interface Patterns. Advances in Engineering Software 40, 12 (Dec. 2009), 1231-1241. https://doi.org/10.1016/j. advengsoft.2009.01.024

[62] Lasse Benn Nørregaard, Philip Kaare Løventoft, Erik Frøkjær, Lise Lauritsen, Emilia Clara Olsson, Louise Andersen, Stine Rauff, and Klaus Martiny. 2014 Patient expectations and experiences from a clinical study in psychiatric care using a self-monitoring system. In Proceedings of the 8th Nordic Conference on Human-Computer Interaction (NordiCHI '14). ACM Press, New York, NY, 991-994. https://doi.org/10.1145/2639189.2670258

[63] NAMI: National Alliance on Mental Illness. 2020. Types of Mental Health Professionals. https://www.nami.org/About-Mental-Illness/Treatments/Types-ofMental-Health-Professionals

[64] Laura Orsolini, Michele Fiorani, and Umberto Volpe. 2020. Digital phenotyping in bipolar disorder: Which integration with clinical endophenotypes and biomarkers? International fournal of Molecular Sciences 21, 20, Article 7684 (2020) 20 pages. https://doi.org/10.3390/ijms21207684

[65] Lace Padilla, Matthew Kay, and Jessica Hullman. 2021. Uncertainty visualization. In Wiley StatsRef: Statistics Reference Online. John Wiley \& Sons, Hoboken, NJ, 1-18. https://doi.org/10.1002/9781118445112.stat08296

[66] Justin Petelka, Lucy Van Kleunen, Liam Albright, Elizabeth Murnane, Stephen Voida, and Jaime Snyder. 2020. Being (in)visible: Privacy, transparency, and disclosure in the self-management of bipolar disorder. In Proceedings of the 2020 CHI Conference on Human Factors in Computing Systems (CHI '20). ACM Press, New York, NY, 1-14. https://doi.org/10.1145/3313831.3376573

[67] Enrico Maria Piras. 2019. Beyond self-tracking: Exploring and unpacking four emerging labels of patient data work. Health Informatics fournal 25, 3 (Sept 2019), 598-607. https://doi.org/10.1177/1460458219833121

[68] Matthew Price, Erica K. Yuen, Elizabeth M. Goetter, James D. Herbert, Evan M. Forman, Ron Acierno, and Kenneth J. Ruggiero. 2014. mHealth: A mechanism to deliver more accessible, more effective mental health= care. Clinical Psychology \& Psychotherapy 21, 5 (2014), 427-436. https://doi.org/10.1002/cpp.1855
[69] Rhys Price-Robertson, Angela Obradovic, and Brad Morgan. 2017. Relational recovery: Beyond individualism in the recovery approach. Advances in Mental Health 15, 2 (2017), 108-120. https://doi.org/10.1080/18387357.2016.1243014

[70] Judith Proudfoot, Gordon Parker, Vijaya Manicavasagar, Dusan Hadzi-Pavlovic, Alexis Whitton, Jennifer Nicholas, Meg Smith, and Rowan Burckhardt. 2012. Effects of adjunctive peer support on perceptions of illness control and understanding in an online psychoeducation program for bipolar disorder: A randomised controlled trial. Fournal of Affective Disorders 142, 1-3 (Dec. 2012), 98-105. https://doi.org/10.1016/j.jad.2012.04.007

[71] Paola Rucci, Ellen Frank, Bryan Kostelnik, Andrea Fagiolini, Alan G. Mallinger, Holly A. Swartz, Michael E. Thase, Lori Siegel, Dorothy Wilson, and David J. Kupfer. 2002. Suicide attempts in patients with bipolar I disorder during acute and maintenance phases of intensive treatment with pharmacotherapy and adjunctive psychotherapy. American fournal of Psychiatry 159, 7 (July 2002), 1160-1164. https://doi.org/10.1176/appi.ajp.159.7.1160

[72] Michael J. Salvo. 2001. Ethics of engagement: User-centered design and rhetorical methodology. Technical Communication Quarterly 10, 3 (July 2001), 273-290. https://doi.org/10.1207/s15427625tcq1003_3

[73] Haran Sened, Iftah Yovel, Eran Bar-Kalifa, Reuma Gadassi, and Eshkol Rafaeli. 2017. Now you have my attention: Empathic accuracy pathways in couples and the role of conflict. Emotion 17, 1 (Feb. 2017), 155-168. https://doi.org/10.1037/ emo0000220

[74] Jaime Snyder, Elizabeth Murnane, Caitie Lustig, and Stephen Voida. 2019. Visually encoding the lived experience of bipolar disorder. In Proceedings of the SIGCHI Conference on Human Factors in Computing Systems (CHI '19). ACM, New York, Article 133, 14 pages. https://doi.org/10.1145/3290605.3300363

[75] Stephen Voida, Mark Matthews, Saeed Abdullah, Mengxi (Chrissie) Xi, Matthew Green, Won Jun Jang, Donald Hu, John Weinrich, Prashama Patil, Mashfiqui Rabbi, Tauhidur Rahman, Geri Gay, Ellen Frank, and Tanzeem Choudhury. 2013. MoodRhythm: Tracking and supporting daily rhythms. In Adjunct Proceedings of the 2013 ACM Conference on Pervasive and Ubiquitous Computing (UbiComp '13 Adjunct). ACM Press, New York, NY, 67-70. https://doi.org/10.1145/2494091. 2494111

[76] Evan Wallace and Dylan Field. 2021. Figma. https://www.figma.com

[77] Peter West, Richard Giordano, Max Van Kleek, and Nigel Shadbolt. 2016. The quantified patient in the doctor's office: Challenges \& opportunities. In Proceedings of the 2016 CHI Conference on Human Factors in Computing Systems (CHI '16). ACM Press, New York, NY, USA, 3066-3078. https://doi.org/10.1145/2858036.2858445

[78] Peter West, Max Van Kleek, Richard Giordano, Mark J. Weal, and Nigel Shadbolt. 2018. Common barriers to the use of patient-generated data across clinical settings. In Proceedings of the 2018 CHI Conference on Human Factors in Computing Systems (CHI '18). ACM Press, New York, NY, Article 484, 13 pages. https: //doi.org/10.1145/3173574.3174058 